\title{
Checklist of the pseudoscorpions (Arachnida: Pseudoscorpiones) of Serbia
}

\section{Реестр иожноскорпионов (Arachnida: Pseudoscorpiones) Сербии}

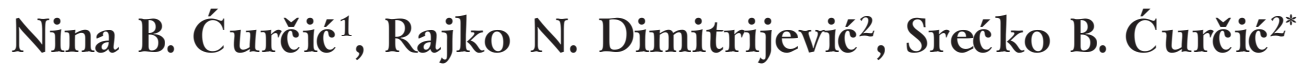 \\ Н.Б. Чурчич ${ }^{1}$, Р.Н. Аимитриевич ${ }^{2}$, С.Б. Чурчич ${ }^{2 *}$
}

\footnotetext{
${ }^{1}$ Geographical Institute "Jovan Cvijić", Serbian Academy of Sciences and Arts, Đure Jakšića 9, 11000 Belgrade, Serbia.

${ }^{2}$ Institute of Zoology, University of Belgrade - Faculty of Biology, Studentski Trg 16, 11000 Belgrade, Serbia. E-mail: srecko@bio.bg.ac.rs
}

KEY WORDS: false scorpions, arthropods, endemics, fauna, diversity, Balkan Peninsula.

КЛЮЧЕВЫЕ СЛОВА: ложноскорпионы, членистоногие, эндемики, фауна, разнообразие, Балканский полуостров.

ABSTRACT. A checklist of all representatives of the order Pseudoscorpiones (Arachnida) from Serbia is provided, based on the available literature. A total of 76 species belonging to 12 genera and four families is recorded in Serbia. Eight species lack precise locality data. General and local distributions of each species are reported. Notes on identification changes and comments on some old records are given for certain species.

How to cite this article: Ćurčić N.B., Dimitrijević R.N., Curčić S.B. 2020. Checklist of the pseudoscorpions (Arachnida: Pseudoscorpiones) of Serbia // Arthropoda Selecta. Vol.29. No.1. P.13-27. doi: 10.15298/ arthsel. 29.1.02

РЕЗЮМЕ. На основании доступной литературы дан реестр всех представителей отряда Pseudoscorpiones (Arachnida) Сербии. В Сербии отмечено 76 видов, принадлежащих к 12 родам и четырем семействам. Для восьми видов нет точных данных о местонахождениях. Для каждого вида даны сведения по общему распространению и находках в регионе. Для некоторых видов даны уточнения по определениям и комментарии по старым находкам.

\section{Introduction}

Small arthropods with a cosmopolitan distribution, pseudoscorpions resemble little scorpions, but lack the metasoma and sting, and are considered close relatives to sun spiders (Solifugae) [Ćurčić et al., 2004; Harvey, 2013]. Currently, this order includes around 3,800 species and subspecies grouped into 27 families [Harvey, 2013]. The number of recorded taxa varies from country to country. Due to insufficient investigations, a low number of pseudoscorpion taxa is known for some countries (e.g., Uddström, Rinne, 2014; Ćurčić, Dimitrijević, 2016; Kolesnikov et al., 2019). According to Harvey [2007, 2013], the countries with the largest numbers of recorded species are the USA (424 spe- cies), Italy (226), Spain (213), Australia (179), Brazil (165), Mexico (161), India (160), South Africa (135), Kenya (133) and France (124). In the Balkan countries, the highest numbers of species have been recorded in Greece (122) and Croatia (112) [Ozimec, 2004; Harvey, 2013].

Taxonomic, faunistic and biogeographical studies of the pseudoscorpion fauna of the Balkan Peninsula have a long tradition. The first pseudoscorpion taxon described from the Balkans was found in Postojna Cave in Slovenia. It was described in 1847 as Blothrus spelaeus Schiødte, 1847, and was later transferred to Neobisium (Blothrus) spelaeum. At that time, there were only a few native arachnologists (Ferdinand Joseph Schmidt, Eugen Adolf Jurinac, Rikard Gasperini), and occasional investigations of the pseudoscorpion fauna were mostly conducted by foreign researchers. During the 19th century and at the beginning of the 20th century, the main contributions to the study of Balkan pseudoscorpions were made by the Hungarian zoologists Ödön Tömösváry and Jenő Daday, the French arachnologist Eugène Simon, the Norwegian zoologist Edvard Ellingsen and the Italian entomologist Giuseppe Müller [Ćurčić et al., 2004]. In 1929, the Serbian zoologist Jovan Hadži, who was born in Romania and worked in the former Yugoslavia (now Slovenia), described a new species from southern Serbia (now the Republic of North Macedonia) - Neobisium (Blothrus) karamani (Hadži, 1929). His studies resulted in the establishment of a number of new pseudoscorpion taxa: one genus, 23 species and five subspecies [Hadži, 1930a, b, 1933a, b, c]. During that period, the Austrian arachnologist Max Beier also made a significant contribution to the knowledge of Balkan pseudoscorpions, describing one genus, 55 species and 24 subspecies in the families Chthoniidae and Neobisiidae [Beier, 1939]. During the 1960s and 1970s, the studies of Jovan Hadži and personal contact with him inspired the Serbian arachnologist Božidar P.M. Ćurčić to begin studies of epigean, endogean and cave-dwelling pseudoscorpions 
in Serbia and other Balkan countries. His systematic investigations resulted in the establishment of six genera and 174 species new to science from the Balkan Peninsula (Serbia, Croatia, Bosnia and Herzegovina, the Republic of North Macedonia, Montenegro, Albania, Greece, Romania, Bulgaria) [Dimitrijević, 2016]. In studying the Serbian pseudoscorpion fauna, Božidar Ćurčić alone or together with his collaborators described one genus and 53 species new to science over a period of 42 years (1972-2013), i.e., 69\% of the total number of pseudoscorpion species recorded in the country.

The Republic of Serbia is situated in Southeastern Europe and lies in the central part of the Balkan Peninsula, where three large tectonic units meet - the Dinaric mountain system, the Carpatho-Balkan mountain system and the Rhodopian mass. It covers an area of $88,499 \mathrm{~km}^{2}$ [Gavrilović, 2017] and is bounded by Hungary in the north, Romania and Bulgaria in the east, the Republic of North Macedonia and Albania in the south, and Croatia, Bosnia and Herzegovina, and Montenegro in the west. According to the Nomenclature of Statistical Territorial Units (NSTJ) classification, the territory of the Republic of Serbia includes five units: Belgrade, Vojvodina, Šmadija and western Serbia, southern and eastern Serbia, and the UN-administered territory of Kosovo and Metohija [Vuković et al., 2011; Gavrilović, 2017].

The comprehensive checklist of Harvey [2013] in- corporates the Serbian fauna and from it some data for Serbia can be segregated. Certain checklists [Ćurčić, 1974; Dimitrijević, 2000] and faunal monographs [Ćurčić et al., 2004] include records for Serbia, but are now outdated.

The aims of this study are twofold: (i) to summarize the current pseudoscorpion fauna in Serbia in the form of a complete checklist containing data on the distributions of taxa both in the world and in Serbia; and (ii) to indicate taxa with unreliable records of presence in the country. The study facilitates access to data on the distribution of pseudoscorpions both in Serbia and on the Balkan Peninsula. We list below 76 species, belonging to 12 genera and four families (taking into account doubtful citations), and include information about their distribution. For certain species, notes on identification changes and comments on some old records are added.

\section{Material and methods}

We collected all available data published on Serbian pseudoscorpions, including records from the UN-administered territory of Kosovo. Dubious findings of pseudoscorpions in the country are marked with an asterisk in the checklist. A table of localities mentioned in the list with their geographical coordinates and altitude is provided (Table 1).

Table 1. List of precise localities of pseudoscorpions from Serbia. Таблица 1. Список местонахождений ложноскорпионов Сербии.

\begin{tabular}{|l|l|l|}
\hline Locality & \multicolumn{1}{|c|}{ Coordinates (N, E) } & \multicolumn{1}{|c|}{ Altitude (m, a.s.l.) } \\
\hline $\begin{array}{l}\text { Aleksići, village of Vrmbaje, } \\
\text { Pridvorica, near Ivanjica }\end{array}$ & $43^{\circ} 28^{\prime} 29.68^{\prime \prime}, 20^{\circ} 23^{\prime} 20.28^{\prime \prime}$ & 877 \\
\hline $\begin{array}{l}\text { An unnamed cave, above the source } \\
\text { of the Vrelo River, Perućac, near } \\
\text { Bajina Bašta }\end{array}$ & $43^{\circ} 57^{\prime} 24.19^{\prime \prime}, 19^{\circ} 25^{\prime} 35.37^{\prime \prime}$ & 243 \\
\hline $\begin{array}{l}\text { An unnamed cave, left bank of the } \\
\text { Timok River, village of Kalna, near } \\
\text { Knjaževac }\end{array}$ & $43^{\circ} 25^{\prime} 25.00^{\prime \prime}, 22^{\circ} 25^{\prime} 10.50^{\prime \prime}$ & 360 \\
\hline $\begin{array}{l}\text { An unnamed cave, Mt. Stol, near } \\
\text { Bor }\end{array}$ & $44^{\circ} 10^{\prime} 16.53^{\prime \prime}, 22^{\circ} 08^{\prime} 13.38^{\prime \prime}$ & 1,034 \\
\hline $\begin{array}{l}\text { An unnamed cave, village of } \\
\text { Pričevići, near Valjevo }\end{array}$ & $44^{\circ} 16^{\prime} 53.57^{\prime \prime}, 19^{\circ} 46^{\prime} 37.49^{\prime \prime}$ & 317 \\
\hline Babin Zub peak, Stara Planina Mts. & $43^{\circ} 22^{\prime} 12.01^{\prime \prime}, 22^{\circ} 36^{\prime} 42.60^{\prime \prime}$ & 1,741 \\
\hline Belgrade & $44^{\circ} 48^{\prime} 12.49^{\prime \prime}, 20^{\circ} 27^{\prime} 38.45^{\prime \prime}$ & 116 \\
\hline Belgrade vicinity & $44^{\circ} 38^{\prime} 22.83^{\prime \prime}, 20^{\circ} 24^{\prime} 08.72^{\prime \prime}$ & 232 \\
\hline $\begin{array}{l}\text { Bogovinska Pećina Cave, village of } \\
\text { Bogovina, near Boljevac }\end{array}$ & $43^{\circ} 53^{\prime} 49.65^{\prime \prime}, 21^{\circ} 55^{\prime} 31.36^{\prime \prime}$ & 291 \\
\hline $\begin{array}{l}\text { Božja Vrata Cave, village of } \\
\text { Beloinje, near Svrljig }\end{array}$ & $43^{\circ} 23^{\prime} 27.72^{\prime \prime}, 22^{\circ} 09^{\prime} 55.77^{\prime \prime}$ & 414 \\
\hline $\begin{array}{l}\text { By the Donje Jezero Lake, valley of } \\
\text { the Grza River, near Paraćin }\end{array}$ & $43^{\circ} 53^{\prime} 43.34^{\prime \prime}, 21^{\circ} 38^{\prime} 36.61^{\prime \prime}$ & 414 \\
\hline $\begin{array}{l}\text { By the Gradac River, village of } \\
\text { Bogatić, near Valjevo }\end{array}$ & $44^{\circ} 12^{\prime} 09.64^{\prime \prime}, 19^{\circ} 50^{\prime} 39.40^{\prime \prime}$ & 459 \\
\hline
\end{tabular}


Table 1 (continued). Таблица 1 (продолжение).

\begin{tabular}{|c|c|c|}
\hline Locality & Coordinates (N, E) & Altitude (m, a.s.l.) \\
\hline $\begin{array}{l}\text { By the Ivanštica River, resort } \\
\text { settlement of Grza, near Paraćin }\end{array}$ & $43^{\circ} 53^{\prime} 50.70^{\prime \prime}, 21^{\circ} 38^{\prime} 51.30^{\prime \prime}$ & 424 \\
\hline $\begin{array}{l}\text { By the Šoferska Noć restaurant, near } \\
\text { the village of Rujište, Mt. Rtanj }\end{array}$ & $43^{\circ} 43^{\prime} 23.11^{\prime \prime}, 21^{\circ} 59^{\prime} 25.75^{\prime \prime}$ & 547 \\
\hline $\begin{array}{l}\text { By the Velika Pećina Cave, village } \\
\text { of Rti, near Kotraža }\end{array}$ & $43^{\circ} 44^{\prime} 09.82^{\prime \prime}, 20^{\circ} 14^{\prime} 55.56^{\prime \prime}$ & 394 \\
\hline $\begin{array}{l}\text { Close to the Kovačevića Pećina } \\
\text { Cave, village of Cerova, near } \\
\text { Krupanj }\end{array}$ & $44^{\circ} 23^{\prime} 40.82^{\prime \prime}, 19^{\circ} 22^{\prime} 55.78^{\prime \prime}$ & 395 \\
\hline $\begin{array}{l}\text { Crni Kamen Cave, village of } \\
\text { Dimce, near Kačanik }\end{array}$ & $42^{\circ} 13^{\prime} 39.06^{\prime \prime}, 21^{\circ} 15^{\prime} 25.18^{\prime \prime}$ & 479 \\
\hline $\begin{array}{l}\text { Čarapićev Brest, Mt. Avala, near } \\
\text { Belgrade }\end{array}$ & $44^{\circ} 41^{\prime} 44.27^{\prime \prime}, 20^{\circ} 31^{\prime} 16.03^{\prime \prime}$ & 318 \\
\hline Deliblato Sands & $44^{\circ} 54^{\prime} 18.76^{\prime \prime}, 21^{\circ} 06^{\prime} 38.31^{\prime \prime}$ & 163 \\
\hline Devojački Bunar, Deliblato Sands & $44^{\circ} 59^{\prime} 39.20^{\prime \prime}, 20^{\circ} 57^{\prime} 28.40^{\prime \prime}$ & 158 \\
\hline $\begin{array}{l}\text { Đeverica Cave, village of Vlasi, near } \\
\text { Dimitrovgrad }\end{array}$ & $42^{\circ} 59^{\prime} 55.04^{\prime \prime}, 22^{\circ} 37 ' 58.93^{\prime \prime}$ & 505 \\
\hline $\begin{array}{l}\text { Fuša Špela Cave, village of } \\
\text { Đeneral Janković, near Kačanik }\end{array}$ & $42^{\circ} 08^{\prime} 55.87^{\prime \prime}, 21^{\circ} 17^{\prime} 52.55^{\prime \prime}$ & 370 \\
\hline Golema Porica Pit, Mt. Rtanj & $43^{\circ} 46^{\prime} 07.74^{\prime \prime}, 21^{\circ} 52^{\prime} 33.25^{\prime \prime}$ & 1,050 \\
\hline $\begin{array}{l}\text { Gornja Lenovačka Pećina Cave, } \\
\text { village of Lenovac, Mt. Tupižnica, } \\
\text { near Zaječar }\end{array}$ & $43^{\circ} 47^{\prime} 56.36^{\prime \prime}, 22^{\circ} 10^{\prime} 22.58^{\prime \prime}$ & 311 \\
\hline $\begin{array}{l}\text { Goveđa Pećina Cave, village of } \\
\text { Crnoklište, near Bela Palanka }\end{array}$ & $43^{\circ} 14^{\prime} 03.78^{\prime \prime}, 22^{\circ} 27^{\prime} 53.10^{\prime \prime}$ & 376 \\
\hline $\begin{array}{l}\text { In front of the Pećina u Vrelu Cave, } \\
\text { village of Jelovica, Stara Planina } \\
\text { Mts., near Pirot }\end{array}$ & $43^{\circ} 10^{\prime} 54.90^{\prime \prime}, 22^{\circ} 49^{\prime} 47.90^{\prime \prime}$ & 757 \\
\hline Jelašnica Gorge, near Niš & $43^{\circ} 16^{\prime} 48.01^{\prime \prime}, 22^{\circ} 03^{\prime} 52.56^{\prime \prime}$ & 350 \\
\hline $\begin{array}{l}\text { Jevremovac Botanical Garden, } \\
\text { Belgrade }\end{array}$ & $44^{\circ} 48^{\prime} 57.16^{\prime \prime}, 20^{\circ} 28^{\prime} 24.30^{\prime \prime}$ & 98 \\
\hline Košutnjak Park, Belgrade & $44^{\circ} 46^{\prime} 02.70^{\prime \prime}, 20^{\circ} 26^{\prime} 07.21^{\prime \prime}$ & 137 \\
\hline $\begin{array}{l}\text { Kršikuće Cave, village of Ugao, near } \\
\text { Tutin }\end{array}$ & $43^{\circ} 03^{\prime} 01.09^{\prime \prime}, 20^{\circ} 02^{\prime} 59.02^{\prime \prime}$ & 1,332 \\
\hline $\begin{array}{l}\text { Kulina peak, Mt. Medvednik, near } \\
\text { Mionica }\end{array}$ & $44^{\circ} 12^{\prime} 35.01^{\prime \prime}, 19^{\circ} 38^{\prime} 20.81^{\prime \prime}$ & 1,222 \\
\hline Lepterija, near Sokobanja & $43^{\circ} 38^{\prime} 12.54^{\prime \prime}, 21^{\circ} 53^{\prime} 15.90^{\prime \prime}$ & 322 \\
\hline $\begin{array}{l}\text { Ludi Vir Cave, village of Krivi Vir, } \\
\text { near Boljevac }\end{array}$ & $43^{\circ} 49^{\prime} 33.44^{\prime \prime}, 21^{\circ} 45^{\prime} 05.17^{\prime \prime}$ & 410 \\
\hline $\begin{array}{l}\text { Lukića Pećina Cave, village of } \\
\text { Lipenović, near Krupanj }\end{array}$ & $44^{\circ} 22^{\prime} 13.00^{\prime \prime}, 19^{\circ} 23^{\prime} 08.89^{\prime \prime}$ & 303 \\
\hline $\begin{array}{l}\text { Manastirska Pećina } 1 \text { Cave, by the } \\
\text { Suvodol Monastery, village of } \\
\text { Minićevo, near Zaječar }\end{array}$ & $43^{\circ} 40^{\prime} 59.87^{\prime \prime}, 22^{\circ} 17^{\prime} 32.43^{\prime \prime}$ & 196 \\
\hline $\begin{array}{l}\text { Manastirska Pećina } 2 \text { Cave, by the } \\
\text { Suvodol Monastery, village of } \\
\text { Minićevo, near Zaječar }\end{array}$ & $43^{\circ} 42^{\prime} 00.30^{\prime \prime}, 22^{\circ} 21^{\prime} 09.20^{\prime \prime}$ & 377 \\
\hline $\begin{array}{l}\text { Milkina (= Mitrova) Pećina Cave, } \\
\text { village of Senje, near Ćuprija }\end{array}$ & $43^{\circ} 55^{\prime} 34.67^{\prime \prime}, 21^{\circ} 22^{\prime} 28.62^{\prime \prime}$ & 124 \\
\hline Mt. Avala, near Belgrade & $44^{\circ} 41^{\prime} 21.00^{\prime \prime}, 20^{\circ} 30^{\prime} 58.00^{\prime \prime}$ & 493 \\
\hline $\begin{array}{l}\text { Mt. Fruška Gora, near Sremski } \\
\text { Karlovci }\end{array}$ & $45^{\circ} 09^{\prime} 26.16^{\prime \prime}, 19^{\circ} 43^{\prime} 39.53^{\prime \prime}$ & 441 \\
\hline
\end{tabular}


Table 1 (continued).

Таблица 1 (продолжение).

\begin{tabular}{|c|c|c|}
\hline Locality & Coordinates $(\mathrm{N}, \mathrm{E})$ & Altitude (m, a.s.l.) \\
\hline Mt. Kosmaj, near Mladenovac & $44^{\circ} 28^{\prime} 19.02^{\prime \prime}, 20^{\circ} 34^{\prime} 32.41^{\prime \prime}$ & 508 \\
\hline Mt. Tara & $43^{\circ} 50^{\prime} 53.24^{\prime \prime}, 19^{\circ} 27^{\prime} 34.45^{\prime \prime}$ & 1,530 \\
\hline Near Istok & $42^{\circ} 46^{\prime} 54.26^{\prime \prime}, 20^{\circ} 29^{\prime} 30.97^{\prime \prime}$ & 477 \\
\hline $\begin{array}{l}\text { Ogorelička Pećina Cave, village of } \\
\text { Sićevo, Svrljiške Planine Mts., near } \\
\text { Niš }\end{array}$ & $43^{\circ} 20^{\prime} 28.54^{\prime \prime}, 22^{\circ} 05^{\prime} 01.79^{\prime \prime}$ & 377 \\
\hline $\begin{array}{l}\text { Pećina pod Kapilijama Cave, village } \\
\text { of Štitkovo, Mt. Javor, near Nova } \\
\text { Varoš }\end{array}$ & $43^{\circ} 28^{\prime} 47.80^{\prime \prime}, 19^{\circ} 58^{\prime} 59.40^{\prime \prime}$ & 1,032 \\
\hline $\begin{array}{l}\text { Pećina u Arsovića Kršu Cave, } \\
\text { village of Maskova, Mt. Javor, near } \\
\text { Nova Varoš }\end{array}$ & $43^{\circ} 30^{\prime} 40.65^{\prime \prime}, 20^{\circ} 03^{\prime} 04.70^{\prime \prime}$ & 896 \\
\hline $\begin{array}{l}\text { Pećina u Brdu Kale Cave, village of } \\
\text { Kalna, near Knjaževac }\end{array}$ & $43^{\circ} 24^{\prime} 32.35^{\prime \prime}, 22^{\circ} 25^{\prime} 35.57^{\prime \prime}$ & 398 \\
\hline $\begin{array}{l}\text { Pećina u Kožuvarskoj Glami Cave, } \\
\text { village of Novo Korito, near Zaječar }\end{array}$ & $43^{\circ} 38^{\prime} 20.68^{\prime \prime}, 22^{\circ} 26^{\prime} 38.57^{\prime \prime}$ & 428 \\
\hline $\begin{array}{l}\text { Pećina u Vrelu Cave, village of } \\
\text { Jelovica, Stara Planina Mts., near } \\
\text { Pirot }\end{array}$ & $43^{\circ} 10^{\prime} 54.38^{\prime \prime}, 22^{\circ} 49^{\prime} 47.32^{\prime \prime}$ & 757 \\
\hline $\begin{array}{l}\text { Peć u Brdu Lolinski Rt Cave, } \\
\text { Beženište, village of Gornja } \\
\text { Kamenica, near Svrljig }\end{array}$ & $43^{\circ} 28^{\prime} 00.40^{\prime \prime}, 22^{\circ} 21^{\prime} 37.97^{\prime \prime}$ & 297 \\
\hline $\begin{array}{l}\text { Petnička Pećina Cave, village of } \\
\text { Petnica, near Valjevo }\end{array}$ & $44^{\circ} 14^{\prime} 43.30^{\prime \prime}, 19^{\circ} 56^{\prime} 08.56^{\prime \prime}$ & 202 \\
\hline Petrovaradin vicinity & $45^{\circ} 14^{\prime} 33.82^{\prime \prime}, 19^{\circ} 52^{\prime} 55.12^{\prime \prime}$ & 81 \\
\hline $\begin{array}{l}\text { Poganovo Monastery, village of } \\
\text { Poganovo, near Dimitrovgrad }\end{array}$ & $42^{\circ} 58^{\prime} 47.74^{\prime \prime}, 22^{\circ} 38^{\prime} 14.79^{\prime \prime}$ & 502 \\
\hline $\begin{array}{l}\text { Pripor Cave, village of Resnik, near } \\
\text { Babušnica }\end{array}$ & $43^{\circ} 06^{\prime} 25.22^{\prime \prime}, 22^{\circ} 21^{\prime} 09.04^{\prime \prime}$ & 601 \\
\hline $\begin{array}{l}\text { Rajkova Pećina Cave, near } \\
\text { Majdanpek }\end{array}$ & $44^{\circ} 26^{\prime} 29.18^{\prime \prime}, 21^{\circ} 57^{\prime} 11.43^{\prime \prime}$ & 467 \\
\hline $\begin{array}{l}\text { Ralenovići, village of Bažale, on the } \\
\text { road from Ivanjica to the Studenica } \\
\text { Monastery }\end{array}$ & $43^{\circ} 31^{\prime} 58.51^{\prime \prime}, 20^{\circ} 22^{\prime} 40.06^{\prime \prime}$ & 1,264 \\
\hline Ravana hill, Ivanjica & $43^{\circ} 34^{\prime} 12.37^{\prime \prime}, 20^{\circ} 14^{\prime} 34.88^{\prime \prime}$ & 474 \\
\hline $\begin{array}{l}\text { Ravnište, village of Dubovo, } \\
\text { Žitorađa, near Prokuplje }\end{array}$ & $43^{\circ} 06^{\prime} 48.40^{\prime \prime}, 21^{\circ} 41^{\prime} 42.78^{\prime \prime}$ & 341 \\
\hline $\begin{array}{l}\text { Sesalačka Pećina Cave, village of } \\
\text { Sesalac, near Sokobanja }\end{array}$ & $43^{\circ} 41^{\prime} 54.97^{\prime \prime}, 21^{\circ} 59^{\prime} 16.76^{\prime \prime}$ & 612 \\
\hline Sokobanja & $43^{\circ} 38^{\prime} 15.40^{\prime \prime}, 21^{\circ} 53^{\prime} 15.50^{\prime \prime}$ & 322 \\
\hline Sremčica & $44^{\circ} 40^{\prime} 34.48^{\prime \prime}, 20^{\circ} 23^{\prime} 32.99^{\prime \prime}$ & 225 \\
\hline $\begin{array}{l}\text { Sveta Dupka Cave, village of } \\
\text { Gulenovci, near Dimitrovgrad }\end{array}$ & $43^{\circ} 07^{\prime} 18.06^{\prime \prime}, 22^{\circ} 49^{\prime} 04.11^{\prime \prime}$ & 1,082 \\
\hline $\begin{array}{l}\text { Sveta Voda Cave, between the } \\
\text { villages of Lis and Turica, near Guča }\end{array}$ & $43^{\circ} 48^{\prime} 08.00^{\prime \prime}, 20^{\circ} 12^{\prime} 51.08^{\prime \prime}$ & 328 \\
\hline $\begin{array}{l}\text { Štitkovska Pećina Cave, village of } \\
\text { Štitkovo, Mt. Javor, near Nova } \\
\text { Varoš }\end{array}$ & $43^{\circ} 29^{\prime} 00.04^{\prime \prime}, 19^{\circ} 58^{\prime} 59.79^{\prime \prime}$ & 1,117 \\
\hline Topčider Park, Belgrade & $44^{\circ} 46^{\prime} 40.94^{\prime \prime}, 20^{\circ} 26^{\prime} 19.85^{\prime \prime}$ & 81 \\
\hline $\begin{array}{l}\text { Vaskova Dupka Cave, village of } \\
\text { Lozan, near Svrljig }\end{array}$ & $43^{\circ} 22^{\prime} 05.84^{\prime \prime}, 22^{\circ} 16^{\prime} 30.89^{\prime \prime}$ & 555 \\
\hline $\begin{array}{l}\text { Velika Balanica Cave, village of } \\
\text { Sićevo, near Niš }\end{array}$ & $43^{\circ} 20^{\prime} 15.10^{\prime \prime}, 22^{\circ} 05^{\prime} 04.30^{\prime \prime}$ & 338 \\
\hline
\end{tabular}


Table 1 (continued). Таблица 1 (продолжение).

\begin{tabular}{|c|c|c|}
\hline Locality & Coordinates $(\mathrm{N}, \mathrm{E})$ & Altitude (m, a.s.l.) \\
\hline $\begin{array}{l}\text { Velika Pećina Cave, village of } \\
\text { Donja Držina, near Pirot }\end{array}$ & $43^{\circ} 05^{\prime} 58.19^{\prime \prime}, 22^{\circ} 36^{\prime} 19.42^{\prime \prime}$ & 423 \\
\hline $\begin{array}{l}\text { Village of Adžine Livade, Gledićke } \\
\text { Planine Mts., near Kragujevac }\end{array}$ & $43^{\circ} 54^{\prime} 14.09^{\prime \prime}, 20^{\circ} 52^{\prime} 19.90^{\prime \prime}$ & 534 \\
\hline $\begin{array}{l}\text { Village of Asanovac, Žitorađa, near } \\
\text { Prokuplje }\end{array}$ & $43^{\circ} 08^{\prime} 16.10^{\prime \prime}, 21^{\circ} 38^{\prime} 44.15^{\prime \prime}$ & 728 \\
\hline Village of Bare, near Sjenica & $43^{\circ} 16^{\prime} 27.25^{\prime \prime}, 20^{\circ} 00^{\prime} 11.63^{\prime \prime}$ & 1,004 \\
\hline $\begin{array}{l}\text { Village of Bratljevo, on the road } \\
\text { from Šančevi to Kovilje, near } \\
\text { Ivanjica }\end{array}$ & $43^{\circ} 28^{\prime} 59.74^{\prime \prime}, 20^{\circ} 10^{\prime} 08.77^{\prime \prime}$ & 967 \\
\hline $\begin{array}{l}\text { Village of Crvena Gora, Mt. Javor, } \\
\text { near Ivanjica }\end{array}$ & $43^{\circ} 24^{\prime} 49.39^{\prime \prime}, 20^{\circ} 04^{\prime} 24.86^{\prime \prime}$ & 1,300 \\
\hline $\begin{array}{l}\text { Village of Golijska Reka, Mt. } \\
\text { Golija, near Ivanjica }\end{array}$ & $43^{\circ} 21^{\prime} 18.75^{\prime \prime}, 20^{\circ} 15^{\prime} 18.32^{\prime \prime}$ & 1,417 \\
\hline $\begin{array}{l}\text { Village of Kovačevac, near } \\
\text { Kačanik, Šar Planina Mts. }\end{array}$ & $42^{\circ} 14^{\prime} 33.81^{\prime \prime}, 21^{\circ} 13^{\prime} 58.40^{\prime \prime}$ & 505 \\
\hline $\begin{array}{l}\text { Village of Kumanica, Mt. Golija, } \\
\text { near Ivanjica }\end{array}$ & $43^{\circ} 28^{\prime} 02.23^{\prime \prime}, 20^{\circ} 14^{\prime} 00.25^{\prime \prime}$ & 660 \\
\hline $\begin{array}{l}\text { Village of Močioci, Mt. Mučanj, } \\
\text { near Ivanjica }\end{array}$ & $43^{\circ} 33^{\prime} 26.62^{\prime \prime}, 19^{\circ} 58^{\prime} 01.18^{\prime \prime}$ & 887 \\
\hline Village of Obrež, near Belgrade & $44^{\circ} 44^{\prime} 09.81^{\prime \prime}, 19^{\circ} 58^{\prime} 38.61^{\prime \prime}$ & 75 \\
\hline $\begin{array}{l}\text { Village of Ovčar Banja, Mt. Debela } \\
\text { Gora }\end{array}$ & $43^{\circ} 53^{\prime} 51.02^{\prime \prime}, 20^{\circ} 11^{\prime} 07.61^{\prime \prime}$ & 293 \\
\hline Village of Pazarište, near Novi Pazar & $43^{\circ} 07^{\prime} 51.70^{\prime \prime}, 20^{\circ} 25^{\prime} 12.22^{\prime \prime}$ & 567 \\
\hline $\begin{array}{l}\text { Village of Ravnište, Žitorađa, near } \\
\text { Prokuplje }\end{array}$ & $43^{\circ} 11^{\prime} 20.89^{\prime \prime}, 21^{\circ} 42^{\prime} 44.00^{\prime \prime}$ & 218 \\
\hline $\begin{array}{l}\text { Virovska Pećina Cave, village of } \\
\text { Virovo, near Arilje }\end{array}$ & $43^{\circ} 46^{\prime} 15.34^{\prime \prime}, 20^{\circ} 08^{\prime} 07.26^{\prime \prime}$ & 459 \\
\hline $\begin{array}{l}\text { Zlotska (= Lazareva) Pećina Cave, } \\
\text { village of Zlot, near Bor }\end{array}$ & $44^{\circ} 01^{\prime} 46.01^{\prime \prime}, 21^{\circ} 57^{\prime} 44.44^{\prime \prime}$ & 313 \\
\hline Žitorađa vicinity, near Prokuplje & $43^{\circ} 11^{\prime} 20.89^{\prime \prime}, 21^{\circ} 42^{\prime} 44.00^{\prime \prime}$ & 218 \\
\hline
\end{tabular}

Localities written in bold are from the UN-administered territory of Kosovo.

Полужирным шрифтом выделены находки в Косово.

Checklist of the Pseudoscorpiones of Serbia

\section{Family CHELIFERIDAE Chelifer Geoffroy, 1762}

\section{Chelifer cancroides (Linnaeus, 1758)}

Distribution in Serbia: Belgrade [Ćurčić et al., 2004]; central Serbia, without precise locality [Ćurčić, 1976b]; southern Serbia, without precise locality [Ćurčić, 1976b].

World distribution: widespread in the Holarctic, with fewer records in the Southern Hemisphere [Harvey, 2013, 2014].

\section{Dactylochelifer Beier, 1932}

\section{*2. Dactylochelifer latreillii (Leach, 1817)}

Distribution in Serbia: Serbia, without precise locality [Tömösváry, 1884; Daday, 1889].

World distribution: Europe [Harvey, 2013].
Note. The only two records of this species from Serbia are very old. It is possible that it does not occur in Serbia, and that the old records are misidentifications.

\section{Rhacochelifer Beier, 1932}

*3. Rhacochelifer maculatus (L. Koch, 1873)

Distribution in Serbia: Serbia, without precise locality [Daday, 1889].

World distribution: Mediterranean [Harvey, 2013].

Note. The only record of this species from Serbia is very old. It is possible that it does not occur in Serbia, and that the old record is a misidentification.

\section{Family CHERNETIDAE Allochernes Beier, 1932}

\section{Allochernes balcanicus Hadži, 1938}

Distribution in Serbia: Crni Kamen Cave, village of Dimce, near Kačanik [Beier, 1963]. 
World distribution: Republic of North Macedonia and Serbia [Harvey, 2013].

\section{Chernes Menge, 1855}

\section{*5. Chernes cimicoides (Fabricius, 1793)}

Distribution in Serbia: Serbia, without precise locality [Tömösváry, 1884; Daday, 1889].

World distribution: Europe and the Near East [Harvey, 2013]

Note. The only two records of this species from Serbia are very old. It is possible that it does not occur in Serbia, and that the old records are misidentifications.

\section{Family CHTHONIIDAE Chthonius C.L. Koch, 1843}

6. Chthonius aquasanctae Ćurčić et Rađa, 2011

Distribution in Serbia: Sveta Voda Cave, between the villages of Lis and Turica, near Guča [Curčić et al., 2011c]. 2013]

World distribution: Serbia [Ćurčić et al., 2011c; Harvey,

\section{Chthonius bogovinae Ćurčić, 1972}

Distribution in Serbia: Bogovinska Pećina Cave, village of Bogovina, near Boljevac [Curčić, 1972d].

World distribution: Serbia [Curčić et al., 2004; Harvey, 2013]

\section{Chthonius ischnocheles (Hermann, 1804)}

Distribution in Serbia: Jevremovac Botanical Garden, Belgrade [Ćurčić, Dimitrijević, 1987; Ćurčić et al., 1995]; Mt. Avala, near Belgrade [Ćurčić et al., 1995]; Sremčica [Ćurčić et al. 1995]; Topčider Park, Belgrade [Ćurčić, Dimitrijević, 1987; Poinar, Curčić, 1994; Ćurčić et al., 1995].

World distribution: Europe, Afro-tropical region and Nearctic [Harvey, 2013].

\section{Chthonius iugoslavicus Ćurčić, 1972}

Distribution in Serbia: Sveta Dupka Cave, village of Gulenovci, near Dimitrovgrad [Ćurčić, 1972a]; Velika Balanica Cave, village of Sićevo, near Niš [Ćurčić, 1988]; Velika Pećina Cave, village of Donja Držina, near Pirot [Ćurčić, 1972a].

World distribution: Serbia [Curčić et al., 2004; Harvey, 2013]

\section{Chthonius kosovensis Ćurčić, 2011}

Distribution in Serbia: near Istok [Ćurčić et al., 2011b]. World distribution: Serbia [Ćurčić et al., 2011b].

\section{Chthonius latidentatus Ćurčić, 1972}

Distribution in Serbia: Milkina (= Mitrova) Pećina Cave, village of Senje, near Ćuprija [Ćurčić, 1972a]. 2013]

World distribution: Serbia [Ćurčić et al., 2004; Harvey,

\section{Chthonius lesnik Ćurčić, 1994}

Distribution in Serbia: Peć u Brdu Lolinski Rt Cave, Beženište, village of Gornja Kamenica, near Svrljig [Ćurčić, 1994].

World distribution: Serbia [Ćurčić et al., 2004; Harvey,
13. Chthonius persimilis Beier, 1939

Distribution in Serbia: village of Bare, near Sjenica [Beier, 1939].

World distribution: Serbia [Harvey, 2013].

\section{Chthonius stevanovici Ćurčić, 1986}

Distribution in Serbia: an unnamed cave, left bank of the Timok River, village of Kalna, near Knjaževac [Curčić, 1986].

World distribution: Serbia [Harvey, 2013].

*15. Chthonius tenuis L. Koch, 1873

Distribution in Serbia: Serbia, without precise locality [Ćurčić, 1974].

World distribution: Europe and the Mediterranean [Harvey, 2013].

Note. Gardini [2009a] redescribed this species based on study of the type specimens. It is possible that the old citation indicating the presence in Serbia [Ćurčić, 1974] refers to another species - C. ischnocheles - such as in the case of a number of the old records in Italy [Gardini, 2009a].

\section{Ephippiochthonius Beier, 1930}

\section{Ephippiochthonius bidentatus (Beier, 1939)}

Distribution in Serbia: Petnička Pećina Cave, village of Petnica, near Valjevo [Beier, 1939].

World distribution: Serbia [Harvey, 2013].

\section{Ephippiochthonius kemza (Ćurčić, Lee et} Makarov, 1993)

Distribution in Serbia: Pećina u Kožuvarskoj Glami Cave, village of Novo Korito, near Zaječar [Ćurčić et al., 1993b].

World distribution: Serbia [Harvey, 2013].

18. Ephippiochthonius metohicus (Ćurčić, 2011)

Distribution in Serbia: near Istok [Ćurčić et al., 2011d]. World distribution: Serbia [Ćurčić et al., 2011d]. 1937)

19. Ephippiochthonius microtuberculatus (Hadži,

Distribution in Serbia: village of Kovačevac, near Kačanik, Šar Planina Mts. [Ćurčić, 1976b].

World distribution: Bulgaria, Republic of North Macedonia and Serbia [Harvey, 2013]. 1790)

20. Ephippiochthonius tetrachelatus (Preyssler,

Distribution in Serbia: an unnamed cave, village of Pričevići, near Valjevo [Curčić, 1972c]; Bogovinska Pećina Cave, village of Bogovina, near Boljevac [Đurović, 1998]; eastern Serbia, without precise locality [Ćurčić, 1976b]; Mt. Avala, near Belgrade [Dimitrijević, 1992]; Petrovaradin vicinity [Hadži, 1933c]; Srem, without precise locality [Ćurčić, 1976b]; Šumadija, without precise locality [Ćurčić, 1976b]; Topčider Park, Belgrade [Poinar, Ćurčić, 1994]; western Serbia, without precise locality [Ćurčić, 1976b].

World distribution: Holarctic, Neotropical region and Australian region [Harvey, 2013].

Note. Gardini [2009a] designated and described a male neotype of this species, since the only existing type specimen is believed to have been lost or destroyed. It is possible that more than one species are confused under E. tetrachelatus [Harvey, 1987; Gardini, 2009b], which might be true for 
certain records from Serbia, especially the older ones [Hadži, 1933c; Ćurčić, 1972c, 1976b].

\section{Ephippiochthonius timacensis (Ćurčić et Sto-} janović, 2012)

Distribution in Serbia: by the Donje Jezero Lake, valley of the Grza River, near Paraćin [Ćurčić et al., 2012a].

World distribution: Serbia [Ćurčić et al., 2012a].

\section{Globochthonius Beier, 1931}

22. Globochthonius pancici (Ćurčić, 1972)

Distribution in Serbia: an unnamed cave, above the source of the Vrelo River, Perućac, near Bajina Bašta [Ćurčić, 1972c].

World distribution: Serbia [Harvey, 2013].

\section{Globochthonius polychaetus (Hadži, 1937)}

Distribution in Serbia: village of Kovačevac, near Kačanik, Šar Planina Mts. [Hadži, 1937].

World distribution: Serbia [Harvey, 2013].

24. Globochthonius purgo (Ćurčić, Lee et Makarov, 1993)

Distribution in Serbia: Pećina u Kožuvarskoj Glami Cave, village of Novo Korito, near Zaječar [Ćurčić et al., 1993b].

World distribution: Serbia [Harvey, 2013].

\section{Neobalkanella Ćurčić, 2013}

\section{Neobalkanella psoglavi (Ćurčić, 1990)}

Distribution in Serbia: Manastirska Pećina 1 Cave, by the Suvodol Monastery, village of Minićevo, near Zaječar [Ćurčić, 1990, 2013].

World distribution: Serbia [Ćurčić, 2013; Harvey, 2013].

\section{Family NEOBISIIDAE} Acanthocreagris Mahnert, 1974

\section{Acanthocreagris ludiviri Curčić, 1976}

Distribution in Serbia: Ludi Vir Cave, village of Krivi Vir, near Boljevac [Ćurčić, 1976a].

World distribution: Serbia [Harvey, 2013].

\section{Neobisium Chamberlin, 1930}

27. Neobisium (Blothrus) babusnicae Ćurčić, 1980

Distribution in Serbia: Pripor Cave, village of Resnik, near Babušnica [Ćurčić, 1980a].

World distribution: Serbia [Harvey, 2013].

28. Neobisium (Blothrus) remyi Beier, 1939

Distribution in Serbia: Kršikuće Cave, village of Ugao, near Tutin [Beier, 1939]. 2013].

World distribution: Montenegro and Serbia [Harvey,

29. Neobisium (Blothrus) stankovici Ćurčić, 1972

Distribution in Serbia: Velika Pećina Cave, village of Donja Držina, near Pirot [Ćurčić, 1972b].

World distribution: Serbia [Harvey, 2013].
30. Neobisium (Blothrus) stitkovense Curčić et Dimitrijević, 2003

Distribution in Serbia: Štitkovska Pećina Cave, village of Štitkovo, Mt. Javor, near Nova Varoš [Ćurčić et al., 2003b].

World distribution: Serbia [Harvey, 2013].

31. Neobisium (Neobisium) babinzub Ćurčić, Dimitrijević, Tomić et Mitić, 2007

Distribution in Serbia: Babin Zub peak, Stara Planina Mts. [Ćurčić et al., 2007].

World distribution: Serbia [Harvey, 2013].

32. Neobisium (Neobisium) borense B. Ćurčić, Dimitrijević et N. Ćurčić, 2011

Distribution in Serbia: an unnamed cave, Mt. Stol, near Bor [Ćurčić et al., 2011a].

World distribution: Serbia [Harvey, 2013].

*33. Neobisium (Neobisium) carcinoides (Hermann, 1804)

Distribution in Serbia: western Serbia, without precise locality [Ćurčić, 1976b].

World distribution: Palaearctic and Afro-tropical region [Harvey, 2013].

34. Neobisium (Neobisium) carpaticum Beier, 1935

Distribution in Serbia: Belgrade vicinity [Ćurčić et al., 1999b]; by the Gradac River, village of Bogatić, near Valjevo [Dimitrijević, 2001, 2002]; Čarapićev Brest, Mt. Avala, near Belgrade [Ćurčić, Dimitrijević, 1983a, b, 1984]; Deliblato Sands [Ćurčić, Dimitrijević, 1988b]; Devojački Bunar, Deliblato Sands [Ćurčić, 1989a]; Jevremovac Botanical Garden, Belgrade [Ćurčić, Dimitrijević, 1988a; Ćurčić et al., 1996a, 1999a]; Košutnjak Park, Belgrade [Ćurčić, Dimitrijević, 1981, 1982, 1988b; Ćurčić, 1989a]; Mt. Avala, near Belgrade [Ćurčić, 1980b, 1982b, 1989a; Ćurčić et al., 1981a, b, 1983, 1994b, 1995; Krunić, Curčić, 1981; Curčić, Dimitrijević, 1985b, 1986a, b, 1988b; Dimitrijević, 1985]; Mt. Fruška Gora, near Sremski Karlovci [Ćurčić et al., 1981b, 1983]; Mt. Kosmaj, near Mladenovac [Ćurčić et al., 1981b, 1983]; Petnička Pećina Cave, village of Petnica, near Valjevo [Ćurčić, Dimitrijević, 1997]; Ravnište, village of Dubovo, Zitorađa, near Prokuplje [Zlatković, 1989]; Srem, without precise locality [Ćurčić, 1976b]; Sremčica [Ćurčić, Dimitrijević, 1988b; Curčić, 1989a]; Sumadija, without precise locality [Ćurčić, 1976b]; Topčider Park, Belgrade [Ćurčić, Dimitrijević, 1985b, 1986a, b, 1988b; Dimitrijević, 1985, 1998; Ćurčić, 1989a; Poinar, Curčić, 1994; Ćurčić et al., 1996a]; village of Obrež, near Belgrade [Ćurčić et al., 1994a, 1996b, c, 1999c; Ćurčić, Tomić, 1999]; Zlotska (= Lazareva) Pećina Cave, village of Zlot, near Bor [Ćurčić et al., 1997b].

World distribution: Carpathian Mountains (Poland, Romania, Slovakia and Serbia) [Harvey, 2013].

\section{Neobisium (Neobisium) cephalonicum (Da-} day, 1888)

Distribution in Serbia: Čarapićev Brest, Mt. Avala, near Belgrade [Ćurčić, Dimitrijević, 1985a]; Mt. Avala, near Belgrade [Ćurčić, 1980b, 1982b, 1989b; Krunić, Ćurčić, 1981; Dimitrijević, 1985; Ćurčić, Dimitrijević, 1986a, 1988b; Ćurčić et al., 1995, 1999a]; Šumadija, without precise loca- 
lity [Ćurčić, 1976b]; Topčider Park, Belgrade [Dimitrijević, 1985; Ćurčić, Dimitrijević, 1986a].

World distribution: Balkan Peninsula and the Near East [Harvey, 2013].

*36. Neobisium (Neobisium) crassifemoratum

(Beier, 1928)

Distribution in Serbia: Serbia, without precise locality [Ćurčić, 1974].

World distribution: Central and Eastern Europe, Balkan Peninsula and the Near East [Harvey, 2013].

37. Neobisium (Neobisium) deltshevi B. Ćurčić, Dimitrijević et N. Curčić, 2010

Distribution in Serbia: Sesalačka Pećina Cave, village of Sesalac, near Sokobanja [Ćurčić et al., 2010a].

World distribution: Serbia [Harvey, 2013].

38. Neobisium (Neobisium) doderoi (Simon, 1896)

Distribution in Serbia: Fuša Špela Cave, village of Đeneral Janković, near Kačanik [Hadži, 1937].

World distribution: Mediterranean, Central Europe and the Near East [Harvey, 2013].

*39. Neobisium (Neobisium) erythrodactylum (L. Koch, 1873)

Distribution in Serbia: Serbia, without precise locality [Ćurčić, 1974].

World distribution: Balkan and Apennine Peninsulas, Central and Eastern Europe and the Near East [Harvey, 2013].

40. Neobisium (Neobisium) fuscimanum (C.L. Koch, 1843)

Distribution in Serbia: Čarapićev Brest, Mt. Avala, near Belgrade [Ćurčić, Dimitrijević, 1986a]; Mt. Avala, near Belgrade [Ćurčić, 1980b, 1982b, 1989b; Ćurčić et al., 1981b, 1983; Dimitrijević, 1985; Ćurčić, Dimitrijević, 1986a, 1988b]; Šumadija, without precise locality [Ćurčić, 1976b]; village of Asanovac, Žitorađa, near Prokuplje [Ćurčić, Dimitrijević, 1991].

World distribution: Balkan and Apennine Peninsulas, Central and Eastern Europe and the Near East [Harvey, 2013].

\section{Neobisium (Neobisium) macrodactylum (Da-} day, 1888)

Distribution in Serbia: Čarapićev Brest, Mt. Avala, near Belgrade [Ćurčić, Dimitrijević, 1985a, b]; Mt. Avala, near Belgrade [Curčić, 1980b, 1982b; Krunić, Curčić, 1981; Dimitrijević, 1985; Ćurčić, Dimitrijević, 1986a; Ćurčić et al., 1994b, 1995]; Mt. Fruška Gora, near Sremski Karlovci [Krunić, Ćurčić, 1981]; Šumadija, without precise locality [Ćurčić, 1976b]; Topčider Park, Belgrade [Curčić, Dimitrijević, 1985a, b, 1986a; Dimitrijević, 1985].

World distribution: Balkan Peninsula, Central Europe and the Near East [Harvey, 2013].

\section{Neobisium (Neobisium) rajkodimitrijevici Ćur-} čić, 2006

Distribution in Serbia: Rajkova Pećina Cave, near Majdanpek [Ćurčić, Tomić, 2006].

World distribution: Serbia [Harvey, 2013].
43. Neobisium (Neobisium) sylvaticum (C.L. Koch, 1835)

Distribution in Serbia: Belgrade vicinity [Ćurčić et al., 1999b]; central Serbia, without precise locality [Ćurčić, 1976b]; Mt. Avala, near Belgrade [Ćurčić, 1980b, 1982b, 1989b; Dimitrijević, 1985; Curčić, Dimitrijević, 1986a, 1988b; Ćurčić et al., 1995]; Mt. Fruška Gora, near Sremski Karlovci [Ćurčić et al., 1981a]; northern Serbia, without precise locality [Ćurčić, 1976b]; Topčider Park, Belgrade [Ćurčić, Dimitrijević, 1985a, 1986a, 1988b; Dimitrijević, 1985; Curčić, 1989b]; Vojvodina Province, without precise locality [Ćurčić, 1974].

World distribution: Europe (except its north) and the Near East [Harvey, 2013].

44. Neobisium (Neobisium) tarae Ćurčić, Dimitrijević, Tomić et Mitić, 2007

Distribution in Serbia: Mt. Tara [Ćurčić et al., 2007].

World distribution: Serbia [Harvey, 2013].

\section{Roncus L. Koch, 1873}

45. Roncus bauk Ćurčić, 1991

Distribution in Serbia: Pećina u Brdu Kale Cave, village of Kalna, near Knjaževac [Ćurčić, 1991b].

World distribution: Serbia [Harvey, 2013].

46. Roncus crnobog Ćurčić, 2013

Distribution in Serbia: Ogorelička Pećina Cave, village of Sićevo, Svrljiške Planine Mts., near Niš [Ćurčić et al., 2013].

World distribution: Serbia [Ćurčić et al., 2013].

47. Roncus golijae Ćurčić, 1997

Distribution in Serbia: village of Kumanica, Mt. Golija, near Ivanjica [Ćurčić et al., 1997a].

World distribution: Serbia [Harvey, 2013].

48. Roncus gruiae Ćurčić et Dimitrijević, 2006

Distribution in Serbia: village of Ovčar Banja, Mt. Debela Gora [Ćurčić et al., 2006].

World distribution: Serbia [Harvey, 2013].

49. Roncus ivanjicae B. Ćurčić et S. Ćurčić, 1995

Distribution in Serbia: Aleksići, village of Vrmbaje, Pridvorica, near Ivanjica [Ćurčić et al., 1997a]; by the Velika Pećina Cave, village of Rti, near Kotraža [Ćurčić et al., 1997a]; Ralenovići, village of Bažale, on the road from Ivanjica to the Studenica Monastery [Ćurčić et al., 1997a]; Ravana hill, Ivanjica [Ćurčić, Ćurčić, 1995]; village of Crvena Gora, Mt. Javor, near Ivanjica [Ćurčić et al., 1997a]; village of Golijska Reka, Mt. Golija, near Ivanjica [Ćurčić et al., 1997a]; village of Kumanica, Mt. Golija, near Ivanjica [Ćurčić et al., 1997a]; village of Močioci, Mt. Mučanj, near Ivanjica [Ćurčić et al., 1997a].

World distribution: Serbia [Harvey, 2013].

\section{Roncus ivansticae Ćurčić, 2012}

Distribution in Serbia: by the Ivanštica River, resort settlement of Grza, near Paraćin [Ćurčić et al., 2012d].

World distribution: Serbia [Ćurčić et al., 2012d].

\section{Roncus jarevid Ćurčić, 2013}

Distribution in Serbia: Gornja Lenovačka Pećina Cave, village of Lenovac, Mt. Tupižnica, near Zaječar [Ćurčić et al., 2013]. 
World distribution: Serbia [Ćurčić et al., 2013].

\section{Roncus jarilo Ćurčić, 1991}

Distribution in Serbia: Ravnište, village of Dubovo, Žitorađa, near Prokuplje [Zlatković, 1989; Ćurčić et al., 1991]; village of Asanovac, Žitorađa, near Prokuplje [Ćurčić, 1991a; Ćurčić et al., 1991, 1992b, 1996a]; Žitorađa vicinity, near Prokuplje [Ćurčić et al., 1996a].

World distribution: Serbia [Harvey, 2013].

Note. Zlatković [1989] and Ćurčić et al. [1991] identified the specimens from the villages of Asanovac and Ravnište as Roncus aff. lubricus, but later it was recognized that they belong to a separate species, $R$. jarilo [Ćurčić, 1991a; Ćurčić et al., 2004].

53. Roncus jelasnicae Ćurčić et Dimitrijević, 2009

Distribution in Serbia: Jelašnica Gorge, near Niš [Ćurčić, Dimitrijević, 2009].

World distribution: Serbia [Harvey, 2013].

54. Roncus krupanjensis B. Ćurčić, Rađa, S. Ćurčić et N. Ćurčić, 2010

Distribution in Serbia: close to the Kovačevića Pećina Cave, village of Cerova, near Krupanj [Ćurčić et al., 2010b]. World distribution: Serbia [Harvey, 2013].

\section{Roncus pannonius Ćurčić, Dimitrijević et} Karamata, 1992

Distribution in Serbia: Belgrade vicinity [Ćurčić et al., 1999b]; Čarapićev Brest, Mt. Avala, near Belgrade [Ćurčić, Dimitrijević, 1983b, 1985a]; Mt. Avala, near Belgrade [Ćurčić, 1980b, 1982b, 1989b; Ćurčić, Dimitrijević, 1986a, 1988b; Ćurčić et al., 1994b, 1995, 1999a]; Petnička Pećina Cave, village of Petnica, near Valjevo [Ćurčić, Dimitrijević, 1997]; Topčider Park, Belgrade [Ćurčić, Dimitrijević, 1985a, 1986a; Curčić et al., 1996a, 1999a]; village of Obrež, near Belgrade [Ćurčić, 1992a, 1992c; Ćurčić et al., 1992a, 1994a, 1995, 1996a, b, c; Poinar, Ćurčić, 1994].

World distribution: Serbia [Harvey, 2013].

Note. Ćurčić [1980b, 1982b, 1989b] and Ćurčić \& Dimitrijević [1983b, 1985a, 1986a, 1988b] identified the specimens from Mt. Avala as Roncus lubricus L. Koch, 1873, as did Ćurčić \& Dimitrijević [1985a, 1986a] for the specimens from Topčider Park, but later it was recognized that they belong to a separate species, R. pannonius [Ćurčić, 1992c; Ćurčić et al., 1992a].

56. Roncus pantici Ćurčić et Dimitrijević, 2004

Distribution in Serbia: Pećina pod Kapilijama Cave, village of Štitkovo, Mt. Javor, near Nova Varoš [Ćurčić, Dimitrijević, 2004].

World distribution: Serbia [Harvey, 2013].

\section{Roncus parablothroides Hadži, 1937}

Distribution in Serbia: Manastirska Pećina 1 Cave, by the Suvodol Monastery, village of Minićevo, near Zaječar [Ćurčić, 1982a; Đurović, 1998]; Manastirska Pećina 2 Cave, by the Suvodol Monastery, village of Minićevo, near Zaječar [Đurović, 1998].

World distribution: Balkan Peninsula and the Near East [Harvey, 2013].

\section{Roncus pljakici Ćurčić, 1973}

Distribution in Serbia: Pećina u Vrelu Cave, village of Jelovica, Stara Planina Mts., near Pirot [Ćurčić, 1973].
World distribution: Serbia [Harvey, 2013].

59. Roncus radgost Ćurčić, 2013

Distribution in Serbia: Golema Porica Pit, Mt. Rtanj [Ćurčić et al., 2013].

World distribution: Serbia [Ćurčić et al., 2013].

60. Roncus radji B. Ćurčić, Rađa, S. Ćurčić et N. Ćurčić, 2010

Distribution in Serbia: Lukića Pećina Cave, village of Lipenović, near Krupanj [Ćurčić et al., 2010b].

World distribution: Serbia [Harvey, 2013].

61. Roncus remesianensis Ćurčić, 1981

Distribution in Serbia: Goveđa Pećina Cave, village of Crnoklište, near Bela Palanka [Ćurčić, 1981; Dimitrijević, 1994, 1997].

World distribution: Serbia [Harvey, 2013].

62. Roncus satoi Ćurčić et Dimitrijević, 1994

Distribution in Serbia: village of Pazarište, near Novi Pazar [Ćurčić, Dimitrijević, 1994].

World distribution: Serbia [Harvey 2013].

\section{Roncus sotirovi Ćurčić, 1982}

Distribution in Serbia: Đeverica Cave, village of Vlasi, near Dimitrovgrad [Ćurčić, 1982a].

World distribution: Serbia [Harvey, 2013].

64. Roncus starivlahi Ćurčić et Dimitrijević, 1998

Distribution in Serbia: village of Bratljevo, on the road from Šančevi to Kovilje, near Ivanjica [Ćurčić, Dimitrijević, 1998].

World distribution: Serbia [Harvey, 2013].

\section{Roncus strahor Ćurčić, 1993}

Distribution in Serbia: Poganovo Monastery, village of Poganovo, near Dimitrovgrad [Ćurčić et al., 1993a].

World distribution: Serbia [Harvey, 2013].

66. Roncus sumadijae Ćurčić, 2012

Distribution in Serbia: village of Adžine Livade, Gledićke Planine Mts., near Kragujevac [Ćurčić et al., 2012b].

World distribution: Serbia [Ćurčić et al., 2012b].

\section{Roncus svanteviti Ćurčić, 1992}

Distribution in Serbia: Pećina u Kožuvarskoj Glami Cave, village of Novo Korito, near Zaječar [Ćurčić, 1992b].

World distribution: Serbia [Harvey, 2013].

\section{Roncus svarozici Ćurčić, 1992}

Distribution in Serbia: in front of the Pećina u Vrelu Cave, village of Jelovica, Stara Planina Mts., near Pirot [Ćurčić, 1992b].

World distribution: Serbia [Harvey, 2013].

69. Roncus svetavodae Ćurčić et Dimitrijević, 2002

Distribution in Serbia: Sveta Voda Cave, between the villages of Lis and Turica, near Guča [Ćurčić, Dimitrijević, 2002].

World distribution: Serbia [Harvey, 2013].

70. Roncus talason Ćurčić, Lee et Makarov, 1993

Distribution in Serbia: Vaskova Dupka Cave, village of Lozan, near Svrljig [Ćurčić et al., 1993b]. 
World distribution: Serbia [Harvey, 2013].

\section{Roncus timacensis Ćurčić, 1981}

Distribution in Serbia: Božja Vrata Cave, village of Beloinje, near Svrljig [Ćurčić, 1981; Dimitrijević, 1994, 1997].

World distribution: Serbia [Harvey, 2013].

\section{Roncus tintilin Ćurčić, 1993}

Distribution in Serbia: by the Šoferska Noć restaurant, near the village of Rujište, Mt. Rtanj [Ćurčić et al., 1993a].

World distribution: Serbia [Harvey, 2013].

\section{Roncus trojan Ćurčić, 1993}

Distribution in Serbia: Lepterija, near Sokobanja [Ćurčić et al., 1993a]; Sokobanja [Poinar, Curčić, 1994].

World distribution: Serbia [Harvey, 2013].

\section{Roncus ursi Ćurčić, 2012}

Distribution in Serbia: Kulina peak, Mt. Medvednik, near Mionica [Ćurčić et al., 2012c].

World distribution: Serbia [Ćurčić et al., 2012c].

75. Roncus virovensis Ćurčić et Dimitrijević, 2002

Distribution in Serbia: Virovska Pećina Cave, village of Virovo, near Arilje [Ćurčić, Dimitrijević, 2002].

World distribution: Serbia [Harvey, 2013].

\section{Roncus vitalei Ćurčić, 2003}

Distribution in Serbia: Pećina u Arsovića Kršu Cave, village of Maskova, Mt. Javor, near Nova Varoš [Ćurčić et al., 2003a].

World distribution: Serbia [Harvey, 2013].

\section{Uncertain and old records of previously} poorly known species

A total of seven pseudoscorpion species out of the 76 recorded in Serbia are reported for the country, but represent dubious findings, since the localities where they were found are imprecisely noted in Serbia (with no other data on the exact location of their finding).
These findings are mostly cited from Tömösváry [1884], Daday [1889] and Ćurčić [1974, 1976b] and refer to species with a wider distribution that are most likely present in Serbia. Their presence in the country needs to be verified by further investigations. The Serbian records of $D$. latreillii, $R$. maculatus and $C$. cimicoides are very old and it is possible that they are misidentifications.

Some of the old records for Serbia [Hadži, 1933c; Ćurčić, 1972c, 1974, 1976b] of previously poorly known species (e.g., C. tenuis, E. tetrachelatus) should be verified in the future, since they might refer to another species, as was reported by Gardini [2009a, b], who recently redescribed the aforementioned species.

\section{Discussion}

According to the current checklist, a total of 76 pseudoscorpion species belonging to 12 genera and four families are recorded from Serbia, which is significantly more than in previous studies (28 species according to Ćurčić [1974], 65 species according to Ćurčić et al. [2004], and 70 species according to Harvey [2013]).

In comparison with the total number of contemporary pseudoscorpion families in the world (27) [Harvey, 2013], the number of families recorded in Serbia (four) represents $14.8 \%$. The number of families recorded is lower than that for many Mediterranean countries [Harvey, 2013]. The most speciose pseudoscorpion families in Serbia are Neobisiidae (51 species or $67.1 \%$ ) and Chthoniidae (20 species or $26.3 \%$ ), while the other two recorded families (Cheliferidae and Chernetidae) have notably smaller numbers of species (three or $4.0 \%$ and two or $2.6 \%$, respectively) (Fig. 1).

The family Neobisiidae is represented by three genera in Serbia (Acanthocreagris, Neobisium and Roncus), while the family Chthoniidae is represented by four genera in the country (Chthonius, Ephippiochthonius, Globochthonius and Neobalkanella) [Zaragoza, 2017]. The family Cheliferidae is represented by three

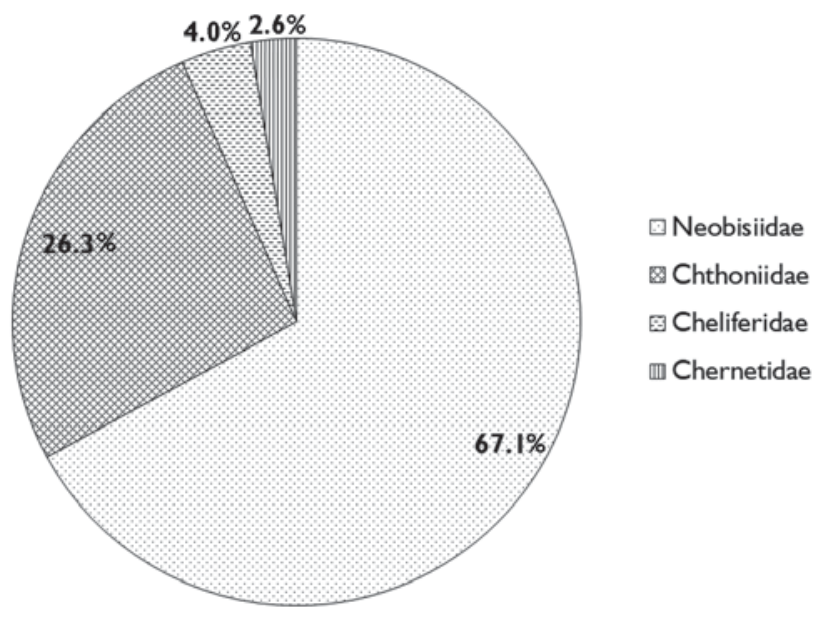

Fig. 1. Composition of Serbian pseudoscorpion species by families.

Рис. 1. Видовой состав фауны ложноскорпионов Сербии по семействам. 


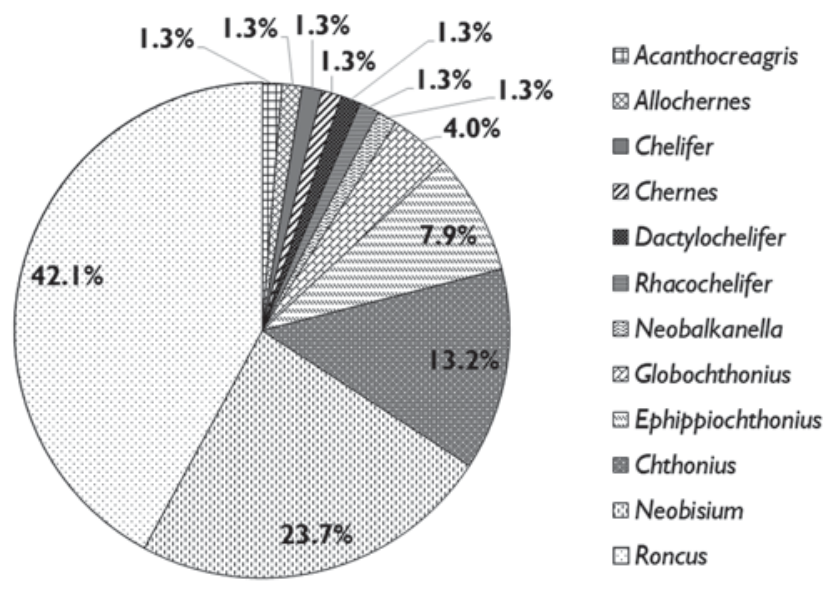

Fig. 2. Composition of Serbian pseudoscorpion species by genera.

Рис. 2. Видовой состав фауны ложноскорпионов Сербии по родам.

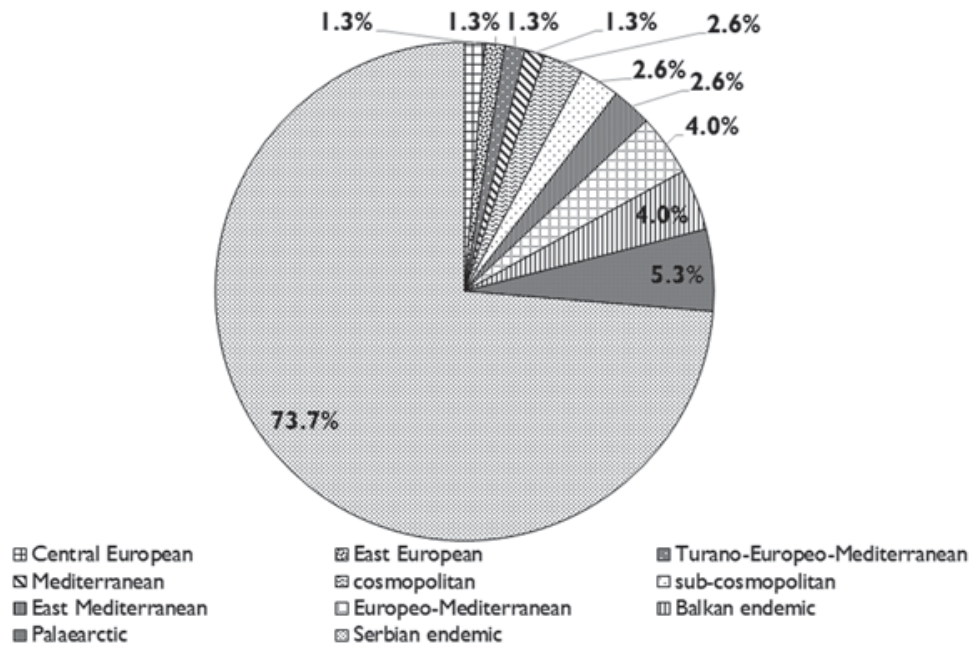

Fig. 3. Zoogeographical composition of Serbian pseudoscorpion fauna (at species level).

Рис. 3. Зоогеографический состав фауны ложноскорпионов Сербии (на видовом уровне).

genera in Serbia (Chelifer, Dactylochelifer and Rhacochelifer), while the family Chernetidae is represented by two genera in the country (Allochernes and Chernes). The most speciose pseudoscorpion genera in Serbia are Roncus (32 species or 42.1\%), Neobisium (18 species or $23.7 \%$ ), Chthonius (10 species or $13.2 \%$ ), Ephippiochthonius (six species or $7.9 \%$ ) and Globochthonius (three species or $4.0 \%$ ), while all other genera (Acanthocreagris, Allochernes, Chelifer, Chernes, Dactylochelifer, Neobalkanella and Rhacochelifer) are represented with only one species or $1.3 \%$ each (Fig. 2).

As for the distribution of pseudoscorpions in Serbia, the greatest number of species inhabit the western, southwestern, eastern and southeastern parts of the country, i.e., the areas with well-developed karst relief, which have been best studied. The territory of Serbia has not been evenly investigated, and the least studied regions are the northern (Vojvodina Province), central and southern (including Kosovo and Metohija Province) parts of Serbia. Out of a total of 76 species, 35 are subterranean, 32 are epigean, four are endogean, three are mostly epigean (with one record from caves), one is troglophilic and one is synanthropic.

All recorded pseudoscorpion species in Serbia can be grouped into 11 zoogeographical categories: cosmopolitan, sub-cosmopolitan, Palaearctic, Central European, East European, Turano-Europeo-Mediterranean, Europeo-Mediterranean, Mediterranean, East Mediterranean, Balkan endemic and Serbian endemic species [Vigna Taglianti et al., 1992, 1999] (Fig. 3). Serbian endemic species are dominant and the most abundant (56 species or $73.7 \%$ ), followed by Palaearctic species (four species or 5.3\%); Europeo-Mediterranean and Balkan endemic species (three species or $4.0 \%$ each); cosmopolitan, East Mediterranean and subcosmopolitan species (two species or $2.6 \%$ each); and 
Central European, East European, Mediterranean and Turano-Europeo-Mediterranean species (one species or $1.3 \%$ each).

Out of the total number of pseudoscorpion species recorded in Serbia, 56 are endemic to the country, three to the Balkan Peninsula and one to the Carpathian Mountains. The family Neobisiidae has the most endemics (with 40 Serbian, one Balkan and one Carpathian endemic species), and within it the genus Roncus is the richest in endemics (containing 31 Serbian endemic species), followed by the genera Neobisium (with eight Serbian, one Balkan and one Carpathian endemic species) and Acanthocreagris (with one Serbian endemic species). Within the family Chthoniidae, 17 endemics were identified (16 Serbian and one Balkan endemic species) within the genera Chthonius (containing eight Serbian endemic species), Ephippiochthonius (with four Serbian and one Balkan endemic species), Globochthonius (with three Serbian endemic species) and Neobalkanella (including one Serbian endemic species). Within the family Chernetidae, one Balkan endemic species of the genus Allochernes is reported.

The research by Serbian authors has been devoted almost entirely to the ground and cave fauna, with little attention being paid to other habitats. Even common and widespread species like Cheiridium museorum (Leach, 1817) have not yet been recorded.

\section{Conclusions}

Generally speaking, it can be stated that the pseudoscorpion fauna of Serbia seems to be very rich. The relatively high number of endemics can be explained by the fact that the diverse types of karst relief in the country have shaped the origin and development of the pseudoscorpion fauna [Ćurčić et al., 2004]. After a thorough analysis of the present checklist and the distribution of Serbian pseudoscorpion species, we can say that the Balkan Peninsula is one of the areas with the richest fauna of pseudoscorpions in the world and represents a hot spot of cave biodiversity at the global level.

Acknowledgements. This study was financially supported by the Serbian Ministry of Education, Science and Technological Development (Grants Nos. III47007 and OI173038). We sincerely appreciate the efforts of two colleagues - Dr. Jelena Ćalić and Vojkan Gajović, MSc. who provided us with certain data on localities. We also thank Mr. Raymond Dooley for proofreading the final version of the text and editing the English. This work is dedicated to the memory of Prof. Božidar P.M. Ćurčić, an eminent Serbian zoologist and academician who devoted his 45year-long professional career to the study of these amazing creatures.

\section{References}

Beier M. 1939. Die Höhlenpseudoscorpione der Balkanhalbinsel // Studien aus dem Gebiete der allgemeinen Karstforschung, der wissenschaftlichen Höhlenkunde, der Eiszeitforschung und den Nachbargebieten. Vol.4. P.1-83.

Beier M. 1963. Ordnung Pseudoscorpionidea (Afterskorpione). Bestimmungsbücher zur Bodenfauna Europas. Lieferung 1. Berlin: Akademie-Verlag. $313 \mathrm{~S}$.

Ćurčić B.P.M. 1972a. Deux nouveaux pseudoscorpions habitant des localités souterraines de la péninsule balkanique: Chthonius caecus ingoslavicus n. ssp. et Chthonius bogovinae latidentatus n. ssp. // Bulletin du Muséum d'Histoire naturelle, Belgrade. Vol.27B. P.125-142.

Ćurčić B.P.M. 1972b. Neobisium (Blothrus) stankovici, nouvelle espèce de pseudoscorpions cavernicoles de la Serbie orientale // Fragmenta Balcanica Musei Macedonici Scientiarum Naturalium. Vol.9. No.9. P.85-96.

Ćurčić B.P.M. 1972c. Nouveaux pseudoscorpions cavernicoles de la Serbie et de la Macédoine // Acta Musei Macedonici Scientiarum Naturalium. Vol.12. No.7. P.141-161.

Ćurčić B.P.M. 1972d. Un pseudoscorpion cavernicole nouveau pour la péninsule des Balkans, Chthonius (C.) bogovinae n. sp. (Chthoniidae, Pseudoscorpiones, Arachnida) // Annales de Spéléologie. Vol.27. No.2. P.341-350.

Ćurčić B.P.M. 1973. A new cavernicolous species of the pseudoscorpion genus Roncus L. Koch (Neobisiidae, Pseudoscorpiones, Arachnida) from the Balkan Peninsula // International Journal of Speleology. Vol.5. No.2. P.127-134.

Ćurčić B.P.M. 1974. Catalogus Faunae Jugoslaviae. Arachnoidea. Pseudoscorpiones. Ljubljana: Academia Scientiarum et Artium Slovenica. $34 \mathrm{p}$.

Ćurčić B.P.M. 1976a. Acanthocreagris ludiviri (Neobisiidae, Pseudoscorpiones, Arachnida), a new species of false scorpions from Serbia // Bulletin du Muséum d'Histoire naturelle, Belgrade. Vol.31B. P.159-168.

Ćurčić B.P.M. 1976b. Une contribution à la connaissance de la faune des pseudoscorpions en Serbie // Bulletin du Muséum d'Histoire naturelle, Belgrade. Vol.31B. P.169-184.

Ćurčić B.P.M. 1980a. A new species of cave-dwelling pseudoscorpion from Serbia (Arachnida: Pseudoscorpiones: Neobisiidae) // Senckenbergiana biologica. Vol.60. Nos 3-4. P.249-254.

Ćurčić B.P.M. 1980b. Accidental and teratological changes in the family Neobisiidae (Pseudoscorpiones, Arachnida) // Bulletin of the British Arachnological Society. Vol.5. Pt.1. P.9-15.

Ćurčić B.P.M. 1981. New cave-dwelling pseudoscorpions from Serbia // Bulletin de l'Académie serbe des Sciences et des Arts, Classe des Sciences mathématiques et naturelles, Sciences naturelles. Vol.75. No.21. P.105-114.

Ćurčić B.P.M. 1982a. New and little-known cave pseudoscorpions from Serbia // Revue Arachnologique. T.3. Fasc.4. P.181-189.

Ćurčić B.P.M. 1982b. Postembryonic Development in the Neobisiidae (Pseudoscorpiones, Arachnida) // Monographs, Vol.545, Department of Sciences, No.56. Belgrade: Serbian Academy of Sciences and Arts. 90 p.

Ćurčić B.P.M. 1986. Chthonius (C.) stevanovici (Chthoniidae, Pseudoscorpiones), nova vrsta pseudoskorpija iz istočne Srbije // Académie serbe des Sciences et des Arts, Édition speciales, Classe de Mathématique, de Physique et des Geosciences, Recueil des Rapports du Comité pour le Karst et la Spéléologie, 2, Présidence. Vol.568. No.3. P.141-154.

Ćurčić B.P.M. 1988. On the taxonomic status of Chthonius caecus iugoslavicus Ćurčić, 1972 (Chthoniidae, Pseudoscorpiones) // Fragmenta Balcanica Musei Macedonici Scientiarum Naturalium. Vol.14. No.1. P.1-10.

Ćurčić B.P.M. 1989a. Segmental anomalies in some European Neobisiidae (Pseudoscorpiones, Arachnida) - part I // Acta Arachnologica. Vol.37. No.2. P.77-87.

Ćurčić B.P.M. 1989b. Segmental anomalies in some European Neobisiidae (Pseudoscorpiones, Arachnida) - part II // Acta Arachnologica. Vol.38. No.1. P.1-10.

Ćurčić B.P.M. 1990. Tyrannochthonius psoglavi, a new species of cave pseudoscorpions from the Balkan Peninsula (Chthoniidae, Pseudoscorpiones) // Revue Arachnologique. T.9. Fasc.1. P.1-9.

Ćurčić B.P.M. 1991a. A new species of Roncus L. Koch from Yugoslavia (Pseudoscorpiones, Neobisiidae) // Archives of Biological Sciences, Belgrade. Vol.43. No. 3-4. P.43P-44P. 
Ćurčić B.P.M. 1991b. A new species of the genus Roncus L. Koch, 1873 (Neobisiidae, Pseudoscorpiones) from East Serbia // Mémoires de Biospéologie. Vol.18. P.165-169.

Curčić B.P.M. 1992a. A new species of Roncus L. Koch, 1873 , from southeastern Europe (Pseudoscorpiones, Neobisiidae) // Proceedings of the Entomological Society of Washington. Vol.94. No.4. P.447-453.

Ćurčić B.P.M. 1992b. New and little-known pseudoscorpions of the genus Roncus L. Koch (Neobisiidae, Pseudoscorpiones) from Serbia, Yugoslavia // Bijdragen tot de Dierkunde. Vol.61. No.4. P.237-249.

Curčić B.P.M. 1992c. On the taxonomic position of Roncus lubricus pannonius Ćurčić, Dimitrijević \& Karamata, 1992 (Neobisiidae, Pseudoscorpiones) // Archives of Biological Sciences, Belgrade. Vol.44. Nos 3-4. P.25P-26P.

Ćurčić B.P.M. 1994. Chthonius (C.) lesnik (Chthoniidae, Pseudoscorpiones), a new pseudoscorpion species from Serbia // Mémoires de Biospéologie. Vol.21. P.25-28.

Ćurčić B.P.M. 2013. On two new genera of pseudoscorpions (Pseudoscorpiones: Chthoniidae) from the northern Mediterranean // Acta zoologica bulgarica. Vol.65. No.2. P.151-158.

Ćurčić B.P.M., Ćurčić N.B., Makarov S.E., Dimitrijević R.N. 1994a. Pedal anomalies in Neobisium carpaticum Beier and Roncus pannonius Ćurčić, Dimitrijević and Karamata (Neobisiidae, Pseudoscorpiones), from Yugoslavia // Acta Arachnologica. Vol.43. No.2. P.145-154.

Ćurčić B.P.M., Ćurčić S.B. 1995. Roncus ivanjicae, a new epigean pseudoscorpion from Serbia, Yugoslavia (Pseudoscorpiones, Neobisiidae), with notes on the validity of some diagnostic characters // Acta Arachnologica. Vol.44. No.1. P.17-25.

Ćurčić B.P.M., Ćurčić S.B., Ćurčić N.B., Makarov S.E. 1993a Three new epigean representatives of Roncus L. Koch, 1873 (Neobisiidae, Pseudoscorpiones) from the Balkan Peninsula // Bijdragen tot de Dierkunde. Vol.62. No.4. P.237-248.

Ćurčić B.P.M., Dimitrijević R.N. 1981. On abnormalities of abdominal segmentation in Neobisium carpaticum Beier, 1934 (Neobisiidae, Pseudoscorpiones, Arachnida) // IX Societas Internationalis Entomofaunistica Europae Centralis (SIEEC) (2228 June 1981). Proceedings. Portorož, Yugoslavia. P.18.

Ćurčić B.P.M., Dimitrijević R.N. 1982. On abnormalities of abdominal segmentation in Neobisium carpaticum Beier (Neobisiidae, Pseudoscorpiones, Arachnida) // Revue Arachnologique. T.4. P.143-150.

Ćurčić B.P.M., Dimitrijević R.N. 1983a. Three more examples of abnormal segmentation of the abdomen in Neobisium carpaticum Beier, 1934 (Arachnida: Pseudoscorpiones, Neobisiidae) // Proceedings of the Entomological Society of Washington. Vol.85. No.2. P.362-365.

Ćurčić B.P.M., Dimitrijević R.N. 1983b. Two more examples of sternal anomaly in Neobisium carpaticum Beier and Roncus lubricus L. Koch (Pseudoscorpiones, Arachnida) // X Societas Internationalis Entomofaunistica Europae Centralis (SIEEC) (15-20 August 1983). Verhandlungen. Budapest, Hungary. P.283-285.

Ćurčić B.P.M., Dimitrijević R.N. 1984. An abnormal carapaceabdominal junction in Neobisium carpaticum Beier, 1934 (Neobisiidae, Pseudoscorpiones) // Archives of Biological Sciences, Belgrade. Vol.36. Nos 1-4. P.9P-10P.

Ćurčić B.P.M., Dimitrijević R.N. 1985a. Abdominal deficiencies in four species of the Neobisiidae (Pseudoscorpiones, Arachnida) // Revue Arachnologique. T.6. Fasc.2. P.91-98.

Ćurčić B.P.M., Dimitrijević R.N. 1985b. Anomalije abdominalnih tergita i sternita kod pseudoskorpija // I Congress of Biosystematists of Yugoslavia (12-14 September 1985). Proceedings and Book of Abstracts. Popova Šapka - Tetovo, Yugoslavia. P.90.

Ćurčić B.P.M., Dimitrijević R.N. 1986a. Abnormalities of carapacal and abdominal segmentation in Neobisium Chamberlin (Neobisiidae, Pseudoscorpiones) // X Congreso internacional de aracnología (2-7 September 1986). Actas. Jaca, Spain. P.1723.

Ćurčić B.P.M., Dimitrijević R.N. 1986b. Teratology of abdominal tergites and sternites in Neobisium carpaticum Beier (Neobisi- idae, Pseudoscorpiones) // Mémoires de la Société royale belge d'Entomologie. Vol.33. P.81-84.

Ćurčić B.P.M., Dimitrijević R.N. 1987. Two segmental anomalies in Chthonius ischnocheles (Hermann) (Chthoniidae, Pseudoscorpiones) // Archives of Biological Sciences, Belgrade. Vol.39. Nos 1-4. P.1P-2P.

Ćurčić B.P.M., Dimitrijević R.N. 1988a. Segmental anomalies in Neobisium carpaticum Beier (Neobisiidae, Pseudoscorpiones) from the Botanical Garden in Belgrade, Yugoslavia // Fragmenta Balcanica Musei Macedonici Scientiarum Naturalium. Vol.13. No.15. P.135-150.

Ćurčić B.P.M., Dimitrijević R.N. 1988b. Segmental deficiencies in some Neobisiidae (Pseudoscorpiones, Arachnida) // XI Europäisches Arachnologisches Colloquium (28 August - 2 September 1988). Abhandlungen. Berlin, Germany. P.89-97.

Ćurčić B.P.M., Dimitrijević R.N. 1991. An example of abnormal carapaco-abdominal fusion in Neobisium aff. fuscimanum (Arachnida, Pseudoscorpiones, Neobisiidae) // Journal of Arachnology. Vol.19. No.3. P.225-226.

Ćurčić B.P.M., Dimitrijević R.N. 1994. Roncus satoi, a new pseudoscorpion species from the Balkan Peninsula (Arachnida: Pseudoscorpiones: Neobisiidae) // Acta Arachnologica. Vol.43. No.2. P.123-130

Ćurčić B.P.M., Dimitrijević R.N. 1997. Further report on some little-known pseudoscorpions from Serbia and Montenegro (Neobisiidae, Pseudoscorpiones) // Archives of Biological Sciences, Belgrade. Vol.49. Nos 1-2. P.55-62.

Ćurčić B.P.M., Dimitrijević R.N. 1998. Roncus starivlahi, a new species of endemic pseudoscorpion from Serbia // Archives of Biological Sciences, Belgrade. Vol.50. No.2. P.19P-20P.

Ćurčić B.P.M., Dimitrijević R.N. 2002. On two new cave relict pseudoscorpions: Roncus svetavodae n. sp. and Roncus virovensis n. sp. (Neobisiidae, Pseudoscorpiones), from Southwestern Serbia // Archives of Biological Sciences, Belgrade. Vol.54. Nos 1-2. P.35-42.

Ćurčić B.P.M., Dimitrijević R.N. 2004. On a new endemic pseudoscorpion from West Serbia - Roncus pantici n. sp. (Neobisiidae, Pseudoscorpiones) // Bulletin de l'Académie serbe des Sciences et des Arts, Classe des Sciences mathématiques et naturelles, Sciences naturelles. Vol.128. No.42. P.319-325.

Ćurčić B.P.M., Dimitrijević R.N. 2009. Roncus jelasnicae, a new epigean pseudoscorpion of the genus Roncus L. Koch, 1873, from East Serbia // Archives of Biological Sciences, Belgrade. Vol.61. No.4. P.751-755.

Ćurčić B.P.M., Dimitrijević R.N., Curčić N.B. 2010a. Neobisium deltshevi (Neobisiidae, Pseudoscorpiones), a new endemic cavedwelling pseudoscorpion from East Serbia // Archives of Biological Sciences, Belgrade. Vol.62. No.1. P.191-198.

Ćurčić B.P.M., Dimitrijević R.N., Ćurčić N.B. 2011a. Neobisium borense sp. n. (Pseudoscorpiones: Neobisiidae), a new endemic species from East Serbia // Acta zoologica bulgarica. Vol.63. No.1. P.3-6.

Ćurčić B.P.M., Dimitrijević R.N., Curčić S.B. 2003a. A new cave pseudoscorpion (Neobisiidae: Pseudoscorpiones) from the Balkan Peninsula // Periodicum biologorum. Vol.105. No.2. P.167170.

Ćurčić B.P.M., Dimitrijević R.N., Ćurčić S.B. 2003b. Neobisium stitkovense n. sp. (Neobisiidae, Pseudoscorpiones) - an endemic cave species from southwestern Serbia // Periodicum biologorum. Vol.105. No.4. P.479-487.

Ćurčić B.P.M., Dimitrijević R.N., Giurginca A., Ilie V., Rađa T., Ćurčić S.B., Tomić V.T. 2006. Four new and endemic species of Roncus L. Koch (Neobisiidae, Pseudoscorpiones) from Romania, Serbia and Montenegro // Periodicum biologorum. Vol.108. No.2. P.213-221.

Ćurčić B.P.M., Dimitrijević R.N., Karamata O.S. 1992a. A revision of some species of Roncus L. Koch (Neobisiidae, Pseudoscorpiones) from North America and South Europe // Journal of Arachnology. Vol.20. No.2. P.114-128.

Ćurčić B.P.M., Dimitrijević R.N., Karamata O.S., Lučić L.R. 1991. Segmental anomalies in Roncus aff. lubricus L. Koch, 1873 (Neobisiidae, Pseudoscorpiones), from Yugoslavia // Journal of Arachnology. Vol.19. No.3. P.215-224. 
Ćurčić B.P.M., Dimitrijević R.N., Legakis A. 2004. The Pseudoscorpions of Serbia, Montenegro, and the Republic of Macedonia. Monographs, Vol. 8, Institute of Zoology. BelgradeAthens: Institute of Zoology, Faculty of Biology, University of Belgrade, Hellenic Zoological Society, Committee for Karst and Speleology, Serbian Academy of Sciences and Arts \& Institute for Nature Conservation of the Republic of Serbia. $400 \mathrm{p}$.

Ćurčić B.P.M., Dimitrijević R.N., Lučić L.R. 1992b. An anomaly of trichobothrial pattern in Roncus jarilo Ćurčić (Neobisiidae, Pseudoscorpiones, Arachnida) // The Canadian Entomologist. Vol.124. No.1. P.201-202.

Ćurčić B.P.M., Dimitrijević R.N., Lučić L.R. 1999a. On some pedipalpal chelal deficiencies in Roncus pannonius, Neobisium bernardi, Neobisium carpaticum and Neobisium cephalonicum (Neobisiidae, Pseudoscorpiones) // Archives of Biological Sciences, Belgrade. Vol.51. No.3. P.41P-42P.

Ćurčić B.P.M., Dimitrijević R.N., Makarov S.E., Ćurčić S.B., Tomić V.T., Antić D.Ž., Ilić B.S. 2013. Roncus radgost n. sp., $R$. jarevid n. sp., and R. crnobog n. sp., three new cave dwellers from eastern Serbia // Archives of Biological Sciences, Belgrade. Vol.65. No.2. P.751-760.

Ćurčić B.P.M., Dimitrijević R.N., Makarov S.E., Lučić L.R., Ćurčić S.B. 1996a. Further report on nematode-pseudoscorpion associations // Acta Arachnologica. Vol.45. No.1. P.43-46.

Curčić B.P.M., Dimitrijević R.N., Makarov S.E., Lučić L.R., Karamata O.S. 1997a. New and Little-Known False Scorpions From the Balkan Peninsula, Principally From Caves, Belonging to the Families Chthoniidae and Neobisiidae (Arachnida, Pseudoscorpiones). Monographs, Vol. 2, Institute of Zoology. Belgrade: Institute of Zoology, Faculty of Biology, University of Belgrade. $159 \mathrm{p}$

Ćurčić B.P.M., Dimitrijević R.N., Makarov S.E., Lučić L.R., Karamata O.S., Tomić V.T. 1997b. The Zlot Cave - a unique faunal refuge (Serbia, Yugoslavia) // Archives of Biological Sciences, Belgrade. Vol.49. Nos 3-4. P.29P-30P.

Ćurčić B.P.M., Dimitrijević R.N., Stojanović D.Z. 2012a. A new epigean pseudoscorpion from East Serbia: Chthonius (Ephippiochthonius) timacensis n. sp. (Chthoniidae, Pseudoscorpiones) // Archives of Biological Sciences, Belgrade. Vol.64 No.3. P.1093-1098.

Ćurčić B.P.M., Dimitrijević R.N., Tomić V.T., Ćurčić N.B. 2011b. A new epigean false scorpion (Chthoniidae, Pseudoscorpiones) from Kosovo // Universiteti i Shkodrës "Luigj Gurakuqi”, Buletin Shkencor, Seria e Shkencave të Natyrës. Vol.61. P.99-106.

Ćurčić B.P.M., Dimitrijević R.N., Tomić V.T., Mitić B.M. 2007. Neobisium babinzub sp. n. and Neobisium tarae sp. n. (Neobisiidae, Pseudoscorpiones) from the central Balkan Peninsula (Serbia) // Biologia, Bratislava. Vol.62. No.1. P.78-83.

Ćurčić B.P.M., Dimitrijević R.N., Tomić V.T., Pecelj M., Ilić B.S., Makarov S.E., Ćurčić N.B., Pešić S. 2012b. A new epigean false scorpion: Roncus sumadijae n. sp. (Neobisiidae, Pseudoscorpiones) from the Balkan Peninsula (western Serbia) // Archives of Biological Sciences, Belgrade. Vol.64. No.2. P.703708.

Ćurčić B.P.M., Krunić M.D., Brajković M.M. 1981a. Further records of teratological changes in the Neobisiidae (Arachnida, Pseudoscorpiones) // Bulletin of the British Arachnological Society. Vol.5. Pt.6. P.280-284.

Ćurčić B.P.M., Krunić M.D., Brajković M.M. 1981b. Tergal and sternal anomalies in the Neobisiidae (Pseudoscorpiones, Arachnida) // IX Societas Internationalis Entomofaunistica Europae Centralis (SIEEC) (22-28 June 1981). Proceedings. Portorož, Yugoslavia. P.20.

Ćurčić B.P.M., Krunić M.D., Brajković M.M. 1983. Tergal and sternal anomalies in Neobisium Chamberlin, 1930 (Neobisiidae, Pseudoscorpiones, Arachnida) // Journal of Arachnology. Vol.11. No.2. P.243-250.

Ćurčić B.P.M., Lee V.F., Makarov S.E. 1993b. New and littleknown cavernicolous species of Chthoniidae and Neobisiidae (Pseudoscorpiones, Arachnida) from Serbia // Bijdragen tot de Dierkunde. Vol.62. No.3. P.167-178.
Ćurčić B.P.M., Legg G., Dimitrijević R.N. 1995. Malformations in pseudoscorpions: a review // Archives of Biological Sciences, Belgrade. Vol.47. Nos 3-4. P.77-100.

Ćurčić B.P.M., Lučić L.R., Makarov S.E., Dimitrijević R.N. 1996b. Polymorphism in pseudoscorpions: sclerite changes in Roncus pannonius Ćurčić, Dimitrijević \& Karamata and Neobisium carpaticum Beier (Neobisiidae, Pseudoscorpiones), from Yugoslavia // I Congress of Biologists of Macedonia (18-21 September 1996). Abstracts. Ohrid, Republic of Macedonia. P.126.

Ćurčić B.P.M., Lučić L.R., Makarov S.E., Dimitrijević R.N., Karamata O.S., Ćurčić S.B., Ćurčić N.B., Radovanović J.S. 1996c. Polymorphism in pseudoscorpions. II. Changes of abdominal segmentation pattern in Neobisium carpaticum Beier and Roncus pannonius Ćurčić, Dimitrijević and Karamata (Neobisiidae, Pseudoscorpiones) // Acta Arachnologica. Vol.45. No.2. P.125-134.

Ćurčić B.P.M., Makarov S.E., Ćurčić S.B., Antić D.Ž., Dimitrijević R.N. 2012c. A new soil pseudoscorpion, Roncus ursi $\mathrm{n}$. sp., from western Serbia (Neobisiidae, Pseudoscorpiones) // Archives of Biological Sciences, Belgrade. Vol.64. No.1. P.379384.

Ćurčić B.P.M., Rađa T., Ćurčić S.B., Ćurčić N.B. 2010b. On Roncus almissae n. sp., $R$. krupanjensis $\mathrm{n}$. $\mathrm{sp}$., and $R$. radji $\mathrm{n}$. sp., three new pseudoscorpions (Pseudoscorpiones, Neobisiidae) from Croatia and Serbia, respectively // Archives of Biological Sciences, Belgrade. Vol.62. No.2. P.503-513.

Ćurčić B.P.M., Rađa T., Dimitrijević R.N., Makarov S.E., Milinčić M. 2011c. A new cave pseudoscorpion from Serbia (Pseudoscorpiones, Chthoniidae) // Archives of Biological Sciences, Belgrade. Vol.63. No.4. P.1257-1263.

Ćurčić B.P.M., Rađa T., Dimitrijević R.N., Makarov S.E., Milinčić M., Pecelj M. 2011d. Two new pseudoscorpions from the UN administered province of Kosovo and Croatia // Archives of Biological Sciences, Belgrade. Vol.63. No.1. P.235-244.

Ćurčić B.P.M., Ribarac-Stepić N., Mijatović N.D., Dimitrijević R.N. 1994b. Electrophoretic protein patterns of body extracts and species identification in some pseudoscorpions (Neobisiidae: Pseudoscorpiones: Arachnida) // Archives Internationales de Physiologie, de Biochimie et de Biophysique. Vol.102. No.6. P.293-296.

Ćurčić B.P.M., Stojanović D.Z., Ilić B.S., Ćurčić N.B. 2012d. Roncus ivansticae (Neobisiidae, Pseudoscorpiones), a new epigean species from eastern Serbia // Archives of Biological Sciences, Belgrade. Vol.64. No.1. P.371-377.

Ćurčić B.P.M., Sudhaus W., Karamata O.S., Tomić V.T., Ćurčić S.B. 1999b. A rhabditid nematode phoretic on pseudoscorpions // Archives of Biological Sciences, Belgrade. Vol.51. No.1. P.19P-20P

Ćurčić B.P.M., Tomić V.T. 1999. On some changes of trichobothrial pattern in Neobisium carpaticum Beier (Neobiisidae, Pseudoscorpiones). Part II // Archives of Biological Sciences, Belgrade. Vol.51. No.3. P.45P-46P.

Ćurčić B.P.M., Tomić V.T. 2006. Neobisium rajkodimitrijevici $\mathrm{n}$. sp. (Neobisiidae, Pseudoscorpiones), a new false scorpion from a cave in eastern Serbia // Archives of Biological Sciences, Belgrade. Vol.58. No.2. P.121-124.

Ćurčić B.P.M., Tomić V.T., Karamata O.S. 1999c. On some changes of trichobothrial pattern in Neobisium carpaticum Beier (Neobiisidae, Pseudoscorpiones). Part I // Archives of Biological Sciences, Belgrade. Vol.51. No.3. P.43P-44P.

Ćurčić N.B., Dimitrijević R.N. 2016. Checklist of the pseudoscorpions (Arachnida: Pseudoscorpiones) of Montenegro // Ecologica Montenegrina. Vol.7. P.439-450.

Daday J. 1889. Adatok a Balkán-félsziget álskorpió-faunájának ismeretéhez (Data ad cognitionem Pseudoscorpionum paeninsulae Balkanicae) // Természetrajzi Füzetek. Vol.12. Nos 2-3. P. $80-84$.

Dimitrijević R.N. 1985. Patomorfološka analiza promena u segmentaciji abdomena kod nekih predstavnika porodice Neobisiidae (Pseudoscorpiones, Arachnida). Master's Thesis. Belgrade: Faculty of Science, University of Belgrade. $72 \mathrm{p}$.

Dimitrijević R.N. 1992. The first record of sternal anomalies in Chthonius aff. tetrachelatus (Preyssler) (Chthoniidae, Pseudo- 
scorpiones) // Archives of Biological Sciences, Belgrade. Vol.44. Nos 3-4. P.23P-24P.

Dimitrijević R.N. 1994. Teratološki fenomeni u strukturnoj organizaciji pseudoskorpija // XI Congress of Speleologists of Yugoslavia (4-6 September 1994). Book of Abstracts. Petnica, Yugoslavia. P.13.

Dimitrijević R.N. 1997. Structural anomalies in cave pseudoscorpions of the Balkan Peninsula // Bulletin de l'Académie serbe des Sciences et des Arts, Classe des mathématiques et Sciences naturelles, Sciences naturelles. Vol.637. No.72. P.107-114.

Dimitrijević R.N. 1998. Further example of cheliceral anomaly in Neobisium carpaticum Beier (Neobisiidae, Pseudoscorpiones) // Bulletin du Muséum d'Histoire naturelle, Belgrade. Vol.48B. P.99-106.

Dimitrijević R.N. 2000. A checklist of cave-dwelling pseudoscorpions (Pseudoscorpiones, Arachnida) from Yugoslavia (Serbia and Montenegro) // Mémoires de Biospéologie. Vol.27. P.3740.

Dimitrijević R.N. 2001. Pseudoscorpions as a diet of other animals // Archives of Biological Sciences, Belgrade. Vol.53. Nos 3-4. P.41P-42P.

Dimitrijević R.N. 2002. Pseudoscorpions as a part of a diet of a brown trout (Salmo trutta L.) // Acta zoologica bulgarica. Vol.54. No.2. P.111-112.

Dimitrijević R.N. 2016. Prof. Dr. Božidar Ćurčić (1946-2015). In memoriam // Journal of Geographical Institute "Jovan Cvijić", SASA. Vol.66. No.1. P.179-182.

Đurović P. (ed.). 1998. Speleological Atlas of Serbia. Belgrade: Geographical Institute "Jovan Cvijić", Serbian Academy of Sciences and Arts, Institute for Nature Conservation of Serbia, Faculty of Geography, University of Belgrade \& Faculty of Biology, University of Belgrade. 290 p.

Gardini G. 2009a. Chthonius (C.) delmastroi n. sp. delle Alpi occidentali e del Piemonte e ridescrizione di Chthonius $(C$.) tenuis L. Koch, 1873 e di C. (C.) submontanus Beier, 1963 (Pseudoscorpiones Chthoniidae) // Rivista piemontese di Storia naturale. Vol.30. P.25-51.

Gardini G. 2009b. Neotype fixation and redescription of Chthonius tetrachelatus (Preyssler, 1790), type species of the subgenus Ephippiochthonius Beier, 1930 (Pseudoscorpiones: Chthoniidae) // Klapalekiana. Vol.45. Nos 1-2. P.23-31.

Gavrilović D. (ed.). 2017. Statistical Yearbook of the Republic of Serbia, 2017. Belgrade: Statistical Office of the Republic of Serbia. $481 \mathrm{p}$

Hadži J. 1930a. Prilog poznavanju pećinskih pseudoskorpija // Glas Srpske kraljevske akademije. Vol.67. P.115-147.

Hadži J. 1930b. Prirodoslovna istraživanja sjevernodalmatinskog otočja. I. Dugi i Kornati. Pseudoscorpiones // Prirodoslovna istraživanja Kraljevine Jugoslavije. Vol.16. P.65-79

Hadži J. 1933a. Beitrag zur Kenntnis der Fauna der Höhle Vjetrenica // Bulletin de l'Académie serbe des Sciences et des Arts, Classe des Sciences mathématiques et naturelles, Sciences naturelles. Vol.1. P.49-79.

Hadži J. 1933b. Beitrag zur Kenntnis der Pseudoskorpionen-Fauna des Küstenlandes // Bulletin international de l'Académie yougoslave des Sciences et des Beaux-arts. Vol.27. P.173-199.

Hadži J. 1933c. Prinos poznavanju pseudoskorpijske faune Primorja // Prirodoslovna istraživanja Kraljevine Jugoslavije. Vol.18. P.125-192.

Hadži J. 1937. Pseudoskorpioniden aus Südserbien // Glasnik Skopskog naučnog društva. Vol.17. P.152-156.
Harvey M.S. 1987. The occurrence in Australia of Chthonius tetrachelatus (Preyssler) (Pseudoscorpionida: Chthoniidae) // Australian entomological Magazine. Vol.13. Nos 5-6. P.68-70.

Harvey M.S. 2007. The smaller arachnid orders: diversity, descriptions and distributions from Linnaeus to the present (1758 to 2007) // Z.-Q. Zhang \& W.A. Shear (eds.). Linnaeus Tercentenary: Progress in Invertebrate Taxonomy. Special Issue, Zootaxa 1668. Auckland: Magnolia Press. P.363-380.

Harvey M.S. 2013. Pseudoscorpions of the World. Version 3.0. Western Australian Museum, Perth, online at: http://www. museum.wa.gov.au/catalogues-beta/pseudoscorpions (accessed on 10 December 2018).

Harvey M.S. 2014. A review and redescription of the cosmopolitan pseudoscorpion Chelifer cancroides (Pseudoscorpiones: Cheliferidae) // Journal of Arachnology. Vol.42. No.1. P.86-104.

Kolesnikov V.B., Turbanov I.S., Gongalsky K.B. 2019. Two new species false scorpion genus Ephippiochthonius Beier, 1930 (Arachnida: Pseudoscorpiones: Chthoniidae) from the Western Ciscaucasia, Russia // Arthropoda Selecta. Vol.28. No.1. P.7382

Krunić M.D., Ćurčić B.P.M. 1981. Correlation between the amount of supercooling and hibernation sites in insects and arachnids // Acta entomologica Jugoslavica. Vol.17. Nos 1-2. P.131-135.

Ozimec R. 2004. List of Croatian pseudoscorpion fauna (Arachnida, Pseudoscorpiones) // Natura Croatica. Vol.13. No.4. P.381394.

Poinar G.O. Jr., Ćurčić B.P.M. 1994. Nematode (Nematoda) associates and parasites of pseudoscorpions (Arachnida) // Journal of Invertebrate Pathology. Vol.63. No.1. P.95-96.

Tömösváry Ö. 1884. Adatok az álskorpiók ismeretéhez (Data ad cognitionem Pseudoscorpionum) // Természetrajzi Füzetek. Vol.8. No.1. P.16-27.

Uddström A., Rinne V. 2014. A pseudoscorpion species new to Finland and an updated checklist (Arachnida: Pseudoscorpiones) // Arthropoda Selecta. Vol.23. No.2. P.225-226.

Vigna Taglianti A., Audisio P.A., Belfiore C., Biondi M., Bologna M.A., Carpaneto G.M., De Biase A., De Felici S., Piattella E., Racheli T., Zapparoli M., Zoia S. 1992. Riflessioni di gruppo sui corotipi fondamentali della fauna W-paleartica ed in particolare italiana // Biogeographia. Vol.16. P.159-179.

Vigna Taglianti A., Audisio P.A., Biondi M., Bologna M.A., Carpaneto G.M., De Biase A., Fattorini S., Piattella E., Sindaco R., Venchi A., Zapparoli M. 1999. A proposal for a chorotype classification of the Near East fauna, in the framework of the Western Palearctic region // Biogeographia. Vol.20. P.31-59.

Vuković D., Jovanović A., Zakić N., Vukotić S. 2011. Nomenclature of statistical territorial units: possibilities of application in Serbia // Journal of Geographical Institute "Jovan Cvijić", SASA. Vol.61. No.2. P.11-24.

Zaragoza J.A. 2017. Revision of the Ephippiochthonius complex in the Iberian Peninsula, Balearic Islands and Macaronesia, with proposed changes to the status of the Chthonius subgenera (Pseudoscorpiones, Chthoniidae) // Zootaxa. Vol.4246. No.1. P.1-221.

Zlatković M.R. 1989. Segmentalne anomalije kod pseudoskorpija Roncus i Neobisium (Pseudoscorpiones) iz okoline Žitorađe. Bachelor's Thesis. Belgrade: Faculty of Science, University of Belgrade. $42 \mathrm{p}$. 